\title{
THE
}

4-8-1999

\section{Asymptotic Convergence Rates of Fourier Path Integral Methods}

Maria Eleftheriou

J. D. Doll

E. Curotto

University of Rhode Island

David L. Freeman

University of Rhode Island, dfreeman@uri.edu

Follow this and additional works at: https://digitalcommons.uri.edu/chm_facpubs

Terms of Use

All rights reserved under copyright.

\section{Citation/Publisher Attribution}

Eleftheriou, M., Doll, J. D., Curotto, E., \& David L. Freeman, D. L. (1999). Asymptotic Convergence Rates of Fourier Path Integral Methods. Journal of Chemical Physics, 110(4), 6657-6672. doi: 10.1063/1.478573 Available at: http://dx.doi.org/10.1063/1.478573 


\title{
Asymptotic convergence rates of Fourier path integral methods
}

\author{
Maria Eleftheriou and J. D. Doll \\ Department of Chemistry, Brown University, Providence, Rhode Island 02912 \\ E. Curotto ${ }^{\text {a) }}$ and David L. Freeman ${ }^{\text {b) }}$ \\ Department of Chemistry, University of Rhode Island, Kingston, Rhode Island 02881
}

(Received 13 October 1998; accepted 13 January 1999)

\begin{abstract}
The asymptotic rates of convergence of thermodynamic properties with respect to the number of Fourier coefficients, $k_{\max }$, included in Fourier path integral calculations are derived. The convergence rates are developed both with and without partial averaging for operators diagonal in coordinate representation and for the energy. Properties in the primitive Fourier method are shown to converge asymptotically as $1 / k_{\max }$ whereas the asymptotic convergence rate is shown to be $1 / k_{\max }^{2}$ when partial averaging is included. Properties are shown to converge at the same rate whether full partial averaging or gradient partial averaging is used. The importance of using the proper operator to optimize convergence rates in partial averaging calculations is emphasized. (C) 1999 American Institute of Physics. [S0021-9606(99)51414-1]
\end{abstract}

\section{INTRODUCTION}

Path integrals ${ }^{1}$ are perhaps the most widely used approaches to include quantum contributions in equilibrium statistical mechanical simulations of many-particle systems. In a path integral simulation the system is described by a set of classical variables along with a set of auxiliary variables used to include the quantum fluctuations. Typical auxiliary degrees of freedom are the set of intermediate time points in discretized path integral approaches ${ }^{2,3}$ and the set of Fourier coefficients in Fourier path integral simulations. ${ }^{4,5}$ Independent of which method is used to evaluate the path integrals, a major practical concern is how to limit the number of auxiliary degrees of freedom included. The computational work grows with the numbers of these auxiliary variables, and practical considerations deem it useful to make the number of variables as small as possible.

The number of auxiliary degrees of freedom included in a path integral simulation cannot be truncated arbitrarily. It is necessary to include sufficient variables so that the calculated thermodynamic properties converge to within the statistical uncertainty of a calculation or to the accuracy needed for a particular application. An important consideration is the determination of the size of the set of auxiliary variables needed for the application at hand. There has been previous formal work ${ }^{6-8}$ on the convergence rate of the action with respect to the number of auxiliary variables included. Determinations of the convergence rates of properties have been made by running a series of calculations with increasing numbers of auxiliary variables until the values of the calculated quantities stop changing to within statistical uncertainties. As we shall demonstrate in this work by numerical example, such an approach to truncation is dangerous, because the convergence of properties with respect to the number of auxiliary variables is often not monotonic. A useful approach is to understand the asymptotic convergence characteristics

\footnotetext{
${ }^{a}$ Present address: Department of Chemistry, Beaver College, Glenside, PA 19038.

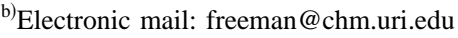

of properties analytically, and truncate only when properties obey the proper asymptotic convergence law. In this work we derive and illustrate numerically such asymptotic convergence rates for thermodynamic properties using the Fourier path integral method.

The contents of the remainder of this paper are as follows. In the next section to establish the notation we review the Fourier path integral method both with and without partial averaging. We also review expressions for the energy estimators used in the Fourier method. In Sec. III we analyze the convergence rates of the errors for properties diagonal in coordinate representation and for the energy both with and without partial averaging. We illustrate the derived asymptotic convergence rates with some model onedimensional potentials in Sec. IV, and in Sec. V we summarize and discuss our results.

\section{THE FOURIER PATH INTEGRAL METHOD}

The Fourier path integral method has been developed and reviewed in previous publications. ${ }^{4,5}$ However, the derivations to follow require careful notation, and we develop the Fourier method here in sufficient detail to establish the necessary notation. For simplicity we restrict the discussion that follows to one-dimensional systems, the extension to many particles in three dimensions being straightforward.

\section{A. The primitive Fourier algorithm}

In path integral simulations for quantum statistical mechanics, the starting point is the Feynman path integral expression for the matrix elements of the density operator at inverse temperature $\beta=1 / k_{B} T$ where $k_{B}$ is the Boltzmann constant

$$
\begin{aligned}
\rho\left(x, x^{\prime} ; \beta\right)= & \left\langle x^{\prime}\left|e^{-\beta \hat{H}}\right| x\right\rangle \\
= & \int D x(\tau) \\
& \times \exp \left(-\frac{1}{\hbar} \int_{0}^{\beta \hbar} d \tau\left\{\frac{1}{2} m \dot{x}^{2}(\tau)+V[x(\tau)]\right\}\right) .
\end{aligned}
$$


In Eq. (1) $\hat{H}$ is the quantum Hamiltonian operator for the system, and in Eq. (2) $\int D x(\tau)$ denotes a summation over all paths connecting the points $x$ to $x^{\prime}$ in the time variable $\tau$. It is useful to define a reduced time variable $u$ defined by $u$ $=\tau / \beta \hbar$ that ranges from 0 to 1 . In any path integral method useful for simulations, the integration over all paths must be transformed into a Riemann integration that can be evaluated on a computer. In the Fourier method the paths are expressed in a Fourier series about some reference path, and the path integral is replaced by Riemann integrals over all the Fourier coefficients. The usual reference path is a straight line path connecting $x$ to $x^{\prime}$ so that the expansion can be represented in a Fourier sine series. An alternative approach ${ }^{9}$ expands the paths about the centroid of the path resulting in an expansion in both sines and cosines. However, Fourier sine series with vanishing end points are known to converge more rapidly than a series containing sines and cosines with no restrictions on the end points, ${ }^{10,11}$ and in this work we restrict attention to expansions about straight line paths. Using the reduced time variable $u$ we write

$$
x(u)=x+\left(x^{\prime}-x\right) u+\sum_{k=1}^{\infty} a_{k} \sin k \pi u .
$$

In any practical calculation the infinite sum is truncated to include $k_{\max }$ Fourier coefficients. We write

$$
x_{k_{\max }}(u)=x+\left(x^{\prime}-x\right) u+\sum_{k=1}^{k_{\max }} a_{k} \sin k \pi u
$$

and

$$
x(u)=x_{k_{\max }}(u)+\delta x(u),
$$

with $\delta x(u)$ representing the summation over the Fourier coefficients whose index exceeds $k_{\max }$; i.e.,

$$
\delta x(u)=\sum_{k=k_{\max }+1}^{\infty} a_{k} \sin k \pi u .
$$

The primitive Fourier expression for the density matrix is obtained by substituting Eq. (4) into Eq. (2). The resulting expression for the density matrix $\rho_{k_{\max }}\left(x, x^{\prime} ; \beta\right)$ can be written conveniently with reference to the free particle density matrix, $\rho_{f p}\left(x, x^{\prime} ; \beta\right)$ as

$$
\frac{\rho_{k_{\max }}\left(x, x^{\prime} ; \beta\right)}{\rho_{f p}\left(x, x^{\prime} ; \beta\right)}=\frac{\int d \mathbf{a} \exp \left[-\Sigma_{k=1}^{k_{\max }} a_{k}^{2} / 2 \sigma_{k}^{2}-\beta \bar{V}_{k_{\max }}\right]}{\int d \mathbf{a} \exp \left[-\Sigma_{k=1}^{k_{\max }} a_{k}^{2} / 2 \sigma_{k}^{2}\right]},
$$

where

$$
\begin{aligned}
& \mathbf{a}=a_{1}, a_{2}, \ldots, a_{k_{\max }} \\
& \rho_{f p}\left(x, x^{\prime} ; \beta\right)=\left(\frac{m}{2 \pi \beta \hbar^{2}}\right)^{1 / 2} \exp \left[-\left(\frac{m}{2 \beta \hbar^{2}}\right)\left(x-x^{\prime}\right)^{2}\right] \\
& \sigma_{k}^{2}=\frac{2 \beta \hbar^{2}}{m(k \pi)^{2}}
\end{aligned}
$$

and the notation $\bar{g}_{k_{\max }}$ represents a path average

$$
\bar{g}_{k_{\max }}=\int_{0}^{1} d u g\left[x_{k_{\max }}(u)\right],
$$

so that

$$
\bar{V}_{k_{\max }}=\int_{0}^{1} d u V\left[x_{k_{\max }}(u)\right] .
$$

Using Eq. (7), expectation values of any operator $f(x)$ that is diagonal in coordinate representation can be obtained

$$
\langle f(x)\rangle_{k_{\max }}=\frac{\int d x d \mathbf{a} \exp \left[-\Sigma_{k=1}^{k_{\max }} a_{k}^{2} / 2 \sigma_{k}^{2}-\beta \bar{V}_{k_{\max }}\right] f(x)}{\int d x d \mathbf{a} \exp \left[-\Sigma_{k=1}^{k_{\max }} a_{k}^{2} / 2 \sigma_{k}^{2}-\beta \bar{V}_{k_{\max }}\right]} .
$$

The true quantum expectation value is given in the limit of infinite $k_{\max }$ so that

$$
\lim _{k_{\max } \rightarrow \infty}\langle f(x)\rangle_{k_{\max }}=\langle f(x)\rangle,
$$

which we write alternatively as $\langle f(x)\rangle_{\infty}$ later in this paper. Expressions for the expectation value of the energy are given in Sec. II D.

\section{B. Limitations and notation}

In the remainder of this paper we limit considerations to potential functions that are continuous with derivatives that are continuous at least through second order. By limiting consideration to such potentials we exclude important systems like hard spheres or sophisticated potentials having conical intersections. The analysis of the convergence rates is also not sufficiently general to include the Coulomb potential even though partial averaging is known ${ }^{12}$ to correct difficulties in path integral treatments that arise from the singular behavior of Coulombic forces at small distances. While these excluded potentials are clearly important, the analysis of their convergence rates requires a development beyond what is presented here. Included in the current analysis are the vast majority of potentials used in modern simulations studies.

For use in the sections that follow, we introduce the notation

$$
\{\mathbf{a}\}=a_{1}, a_{2}, \ldots, a_{k_{\max }}, a_{k_{\max }+1}, \ldots
$$

and

$$
\{\{\mathbf{a}\}\}=a_{k_{\text {max }}+1}, a_{k_{\text {max }}+2}, \ldots .
$$

In other words, a represents the first $k_{\max }$ Fourier coefficients, $\{\mathbf{a}\}$ collectively represents all Fourier coefficients, whereas $\{\{\mathbf{a}\}\}$ represents those Fourier coefficients whose indices exceed $k_{\max }$.

\section{Partial averaging}

Contributions from the portion of the Fourier expansion with Fourier indices greater than $k_{\max }$ can be included in an approximate way using the partial average (pa) method introduced some time ago. ${ }^{5,13,14}$ To develop partial averaging, we express the exact density matrix as a Fourier path integral and separate the integration over the Fourier coefficients into the lower-order a and higher-order $\{\{\mathbf{a}\}\}$ contributions. As 
shown elsewhere, 5,14 the integration over the higher-order coefficients results in the average of an exponential that can be expanded in a series of cumulants, the first term of which is the partial averaged potential. We write the resulting expression for the density matrix by

$$
\frac{\rho_{\mathrm{pa}, k_{\max }}\left(x, x^{\prime} ; \beta\right)}{\rho_{f p}\left(x, x^{\prime} ; \beta\right)}=\frac{\int d \mathbf{a} \exp \left[-\Sigma_{k=1}^{k_{\max }} a_{k}^{2} / 2 \sigma_{k}^{2}-\beta \bar{V}_{\mathrm{pa}, k_{\max }}\right]}{\int d \mathbf{a} \exp \left[-\Sigma_{k=1}^{k_{\max }} a_{k}^{2} / 2 \sigma_{k}^{2}\right]},
$$

where the partial averaged potential is given by the Gaussian transform of the bare potential

$$
V_{\mathrm{pa}}(x, u)=\frac{1}{\sqrt{2 \pi \sigma^{2}(u)}} \int_{-\infty}^{\infty} d p e^{-p^{2} / 2 \sigma^{2}(u)} V(x+p),
$$

with $\sigma^{2}(u)$ the free particle second-order moments of the contribution to the path of the Fourier coefficients that exceed $k_{\text {max }}$; i.e., defined by

$$
\sigma^{2}(u)=\frac{\int d\{\{\mathbf{a}\}\} \exp \left[-\sum_{k=k_{\max }+1}^{\infty} a_{k}^{2} / 2 \sigma_{k}^{2}\right]\left(\sum_{k=k_{\max }+1}^{\infty} a_{k} \sin k \pi u\right)^{2}}{\int d\{\{\mathbf{a}\}\} \exp \left[-\sum_{k=k_{\max }+1}^{\infty} a_{k}^{2} / 2 \sigma_{k}^{2}\right]} .
$$

The second-order moments can be evaluated analytically so that

$$
\sigma^{2}(u)=\frac{\beta \hbar^{2}}{m} u(1-u)-\sum_{k=1}^{k_{\max }} \sigma_{k}^{2} \sin ^{2} k \pi u .
$$

Using the Euler-MacLaurin summation formula, ${ }^{15}$ it can be shown that $\sigma^{2}(u)$ vanishes with increasing $k_{\max }$ as $1 / k_{\max }$ so that

$$
\lim _{k_{\max } \rightarrow \infty}\left(\bar{V}_{\mathrm{pa}, k_{\max }}-\bar{V}_{k_{\max }}\right)=0 .
$$

Expectation values obtained using either the partial averaged potential or the bare potential in the action converge to the same result. As shown in Sec. III, the rate of convergence of the two methods is considerably different.

For some applications, the Gaussian transform of the interaction potential may be either too difficult to evaluate analytically or even be undefined. In such cases the potential in Eq. (18) can be expanded in a power series in $p$ prior to integration resulting in the expression

$V_{\mathrm{pa}}(x, u)=V(x)+\frac{1}{2} \sigma^{2}(u) V^{\prime \prime}(x)+\frac{1}{4 !} \sigma^{4}(u) V^{\prime \prime \prime \prime}(x)+\cdots$.

If Eq. (22) is truncated at second order, the result is called the gradient partial average (gpa) potential

$$
V_{\mathrm{gpa}}(x, u)=V(x)+\frac{1}{2} \sigma^{2}(u) V^{\prime \prime}(x) .
$$

Because $\sigma^{2}(u)$ vanishes for large $k_{\max }$, simulations that use the gradient partial average potential in the action converge to the correct limit. In Sec. III we show the asymptotic rates of convergence of the gradient partial average method and the partial average method are the same.

\section{Energy estimators}

The central quantity needed for the determination of thermodynamic properties is the total energy. If the total energy is known as a function of temperature, the partition function can be obtained by state integration thereby determining all equilibrium thermodynamic properties. Because the Hamiltonian operator is not diagonal in coordinate representation, the construction of energy estimators requires a separate treatment. Here we review the three principal estimators that have been used in the Fourier method. ${ }^{5}$ Although the Hamiltonian is not diagonal in coordinate representation, the estimators can be expressed so that only the diagonal elements of the density matrix are needed in the determination of the expectation values. The last approach discussed in this section, the virial estimator, has not been examined adequately in previous work, and we give the virial estimator a fuller derivation.

\section{The H-method}

The $H$-method estimator is derived by direct operation of the Hamiltonian operator on the density matrix

$$
\langle E\rangle=\frac{\left.\int d x \hat{H} \rho\left(x, x^{\prime} ; \beta\right)\right|_{x=x^{\prime}}}{\int d x \rho(x, x ; \beta)},
$$

which for a finite set of Fourier coefficients leads to the expression $^{5}$

$$
\begin{aligned}
\langle E\rangle_{k_{\max }}= & \left\langle V+\frac{1}{2 \beta}-\frac{(\beta \hbar)^{2}}{2 m}\left(\int_{0}^{1} d u(1-u) V^{\prime}\left[x_{k_{\max }}(u)\right]\right)^{2}\right. \\
& \left.+\frac{\beta \hbar^{2}}{2 m} \int_{0}^{1} d u(1-u)^{2} V^{\prime \prime}\left[x_{k_{\max }}(u)\right]\right\rangle_{k_{\max }} .
\end{aligned}
$$

While the $H$-method has been found to be useful in many applications, Eq. (25) requires two additional $u$-integrations to be evaluated, and the approach can be computationally demanding. Equation (25) is expressed explicitly for the primitive Fourier method. If partial averaging is used, the first and second derivatives of the potential in the last two terms on the right hand side of the equation should be replaced by the first and second derivatives of the effective partial averaged potential.

\section{The T-method}

The $T$-method expression for the energy is obtained by temperature differentiation of the canonical partition function 


$$
\langle E\rangle=-\left(\frac{\partial \ln Q}{\partial \beta}\right),
$$

where

$$
Q(\beta)=\int d x \rho(x, x ; \beta) .
$$

In the primitive Fourier method the resulting expression is

$$
\langle E\rangle_{k_{\max }}=\frac{k_{\max }+1}{2 \beta}+\left\langle\bar{V}_{k_{\max }}-\sum_{k=1}^{k_{\max }} a_{k}^{2} / 2 \beta \sigma_{k}^{2}\right\rangle_{k_{\max }},
$$

and when partial averaging is included we obtain

$$
\langle E\rangle_{k_{\max }}=\frac{k_{\max }+1}{2 \beta}+\left\langle 2 \bar{V}_{\mathrm{pa}, k_{\max }}-\bar{V}_{k_{\max }}-\sum_{k=1}^{k_{\max }} a_{k}^{2} / 2 \beta \sigma_{k}^{2}\right\rangle_{k_{\max }} .
$$

The virtue of both Eqs. (28) and (29) is that the average is taken for quantities that have already been calculated in determining the action. Unlike Eq. (25) where new quantities must be determined at each Monte Carlo point, the $T$-method expressions require no additional overhead. As shown elsewhere, ${ }^{5}$ the statistical error associated with the expressions evaluated using the $T$-method estimator grows as $\sqrt{k_{\max }}$. The origin of the variance difficulties in Eqs. (28) and (29) is the term $k_{\max } / 2 \beta$ whose contribution is canceled by the average over the quadratic form in the Fourier coefficients. As shown in Sec. II D 3, the cancelations are explicitly removed in the virial estimator resulting in a wellbehaved expression for the energy. Applications using the $T$-method can be problematic for calculations requiring many Fourier coefficients.

\section{The virial estimator}

In previous work ${ }^{16}$ we have discussed an energy estimator based on the quantum virial theorem. This estimator has required the evaluation of the gradient of the potential at a point, and as discussed elsewhere ${ }^{16}$ the estimator has proved to be ill-behaved for potentials with strongly repulsive walls. Here we derive an alternate virial estimator that works well for such potentials, and is closely related to virial estimators found to be useful in discretized path integral treatments. ${ }^{2,17}$ The convergence characteristics with respect to $k_{\max }$ of this estimator are identical to the $T$-method estimator but with cancelation of the ill-behaved terms made explicit. The derivation that follows includes partial averaging. We discuss the modifications necessary for the primitive Fourier method after the derivation is complete.

We begin with the expression

$$
\begin{aligned}
& \left\langle\left(x \frac{\partial}{\partial x}+\sum_{k=1}^{k_{\max }} a_{k} \frac{\partial}{\partial a_{k}}\right)\left(\sum_{k=1}^{k_{\max }} a_{k}^{2} / 2 \sigma_{k}^{2}+\beta \bar{V}_{\mathrm{pa}, k_{\max }}\right)\right\rangle_{k_{\max }} \\
& =k_{\max }+1,
\end{aligned}
$$

which can be proved using integration by parts. When integrating by parts, the surface terms resulting from integrations with respect to the Fourier coefficients vanish automatically, but Eq. (30) follows only for potentials where $x \exp \left(-\beta \bar{V}_{\mathrm{pa}, k_{\max }}\right)$ vanishes at the end-points of the integration. If the derivatives in Eq. (30) are performed explicitly, on rearrangement we obtain

$$
\begin{gathered}
\frac{k_{\max }+1}{2 \beta}-\left\langle\sum_{k=1}^{k_{\max }} a_{k}^{2} / 2 \beta \sigma_{k}^{2}\right\rangle_{k_{\max }} \\
=\left\langle\frac{1}{2} x \frac{\partial \bar{V}_{\mathrm{pa}, k_{\max }}}{\partial x}+\frac{1}{2} \sum_{k} a_{k} \frac{\partial \bar{V}_{\mathrm{pa}, k_{\max }}}{\partial a_{k}}\right\rangle_{k_{\max }} .
\end{gathered}
$$

On substitution of Eq. (31) into Eq. (29), the resulting expression for the virial estimator is obtained

$$
\langle E\rangle_{k_{\max }}=\left\langle\frac{1}{2} \overline{x(u) V_{\mathrm{pa}, k_{\max }}^{\prime}[x(u)]}+2 \bar{V}_{\mathrm{pa}, k_{\max }}-\bar{V}_{k_{\max }}\right\rangle_{k_{\max }} .
$$

Equation (32) can be transformed into the appropriate expression for the primitive Fourier method by replacing $\bar{V}_{\mathrm{pa}, k_{\max }}$ by $\bar{V}_{k_{\max }}$ everywhere it appears.

For a system having $N$ particles, care must be taken in applying Eq. (32) if the potential energy is independent of the coordinate of the center of mass. Examples of systems where the potential energy is independent of the center of mass include clusters defined using a constraining potential placed about the center of mass. ${ }^{4,18}$ Clusters have been the subject of many simulation studies using path integral methods. ${ }^{4}$ In such cases the surface term arising from the center of mass motion does not vanish, and as shown in Appendix A the virial estimator gives a kinetic energy contribution that is too small by $1 /(2 \beta)$ in one-dimension and $3 /(2 \beta)$ in three dimensions. In other words, the virial estimator for such systems gives the total energy less the kinetic energy of the center of mass.

\section{ASYMPTOTIC CONVERGENCE RATES}

In this section we derive the asymptotic rates of convergence of thermodynamic properties with respect to the number of Fourier coefficients included in the calculation. The detailed convergence rates can depend on the property, the estimator used, and whether partial averaging is included. To clarify our ultimate conclusions, we begin with an analysis of properties using the primitive Fourier method. We follow with an analysis of partial averaging. In each case we first discuss expectation values of properties that are diagonal in coordinate representation, followed by path averaged properties as discussed in the context of the energy estimators.

\section{A. The primitive Fourier method}

\section{Properties diagonal in coordinate representation}

We begin with the expression for the density [Eq. (7)] and the expression for the expectation value of an operator diagonal in coordinate representation $f(x)$ [Eq. (13)] when $k_{\max }$ Fourier coefficients are included. We define

$$
S_{k_{\max }}(x,\{\mathbf{a}\})=\sum_{k=1}^{\infty} a_{k}^{2} / 2 \sigma_{k}^{2}+\beta \bar{V}_{k_{\max }},
$$

and write 


$$
\langle f(x)\rangle_{k_{\max }}=\frac{\int d x d\{\mathbf{a}\} \exp \left[-S_{k_{\max }}(x,\{\mathbf{a}\})\right] f(x)}{\int d x d\{\mathbf{a}\} \exp \left[-S_{k_{\max }}(x,\{\mathbf{a}\})\right]} .
$$

It is important to recognize that the action defined in Eq. (33) formally depends on the entire infinite set of Fourier coefficients. However, the integration in Eq. (34) over the Fourier coefficients with indices greater than $k_{\max }$ cancel in numerator and denominator, and the expression for the expectation value in Eq. (34) is formally identical to Eq. (13). We next define

$$
\Delta S(x,\{\mathbf{a}\})=S_{\infty}(x,\{\mathbf{a}\})-S_{k_{\max }}(x,\{\mathbf{a}\}),
$$

where $S_{\infty}(x,\{\mathbf{a}\})$ denotes the full action; i.e., the action with the full infinite set of Fourier coefficients included. Using Eqs. (34) and (35), we obtain an expression for the expectation value of $f$ at the limit of an infinite set of Fourier coefficients

$$
\begin{aligned}
\langle f(x)\rangle_{\infty} & =\frac{\int d x d\{\mathbf{a}\} \exp \left[-S_{k_{\max }}(x,\{\mathbf{a}\})\right] e^{-\Delta S} f(x)}{\int d x d\{\mathbf{a}\} \exp \left[-S_{k_{\max }}(x,\{\mathbf{a}\})\right] e^{-\Delta S}} \\
& =\frac{\left\langle e^{-\Delta S} f(x)\right\rangle_{k_{\max }}}{\left\langle e^{-\Delta S}\right\rangle_{k_{\max }}} .
\end{aligned}
$$

As $k_{\max }$ increases, $\Delta S$ becomes small, and it makes sense to expand the exponentials in Eq. (37) in power series

$$
\langle f(x)\rangle_{\infty}=\frac{\left\langle f(x)\left(1-\Delta S+1 / 2(\Delta S)^{2}+\cdots\right\rangle_{k_{\max }}\right.}{\left\langle 1-\Delta S+1 / 2(\Delta S)^{2}+\cdots\right\rangle_{k_{\max }}} .
$$

For $\Delta S$ small the denominator can be expanded in a geometric series. After grouping terms of like powers in $\Delta S$ we obtain

$$
\langle f(x)\rangle_{\infty}=\langle f(x)\rangle_{k_{\max }}+\epsilon
$$

where the error $\epsilon$ is given by a series in increasing fluctuations of $f(x)$ and $\Delta S$

$$
\epsilon=\phi_{1}+\phi_{2}+\cdots
$$

with

$$
\begin{aligned}
\phi_{1}= & \langle\Delta S\rangle_{k_{\max }}\langle f(x)\rangle_{k_{\max }}-\langle\Delta S f(x)\rangle_{k_{\max }}, \\
\phi_{2}= & \frac{1}{2}\left[\left\langle(\Delta S)^{2} f(x)\right\rangle_{k_{\max }}-\langle f(x)\rangle_{k_{\max }}\left\langle(\Delta S)^{2}\right\rangle_{k_{\max }}\right] \\
& -\langle\Delta S\rangle_{k_{\max }}\left[\langle\Delta S f(x)\rangle_{k_{\max }}-\langle\Delta S\rangle_{k_{\max }}\langle f(x)\rangle_{k_{\max }}\right],
\end{aligned}
$$

and so on. The leading term that decays with $k_{\max }$ in the error can be obtained by examining the $k_{\max }$ dependence of $\phi_{1}$. We begin with the expectation value of $\Delta S$ averaged with respect to the $k_{\max }$ dependent distribution

$$
\begin{aligned}
\langle\Delta S\rangle_{k_{\max }}= & \beta\left\langle\bar{V}_{\infty}-\bar{V}_{k_{\max }}\right\rangle_{k_{\max }} \\
= & \beta \int_{0}^{1} d u\left\langle V[x(u)]-V\left[x_{k_{\max }}(u)\right]\right\rangle_{k_{\max }} \\
= & \beta \int_{0}^{1} d u\left\langle V\left[x_{k_{\max }}(u)+\delta x(u)\right]\right. \\
& \left.-V\left[x_{k_{\max }}(u)\right]\right\rangle_{k_{\max }} .
\end{aligned}
$$

We next expand the potential in powers of $\delta x(u)$ to obtain

$$
\begin{aligned}
\langle\Delta S\rangle_{k_{\max }}= & \beta \int_{0}^{1} d u\left\langle\delta x(u) V^{\prime}\left[x_{k_{\max }}(u)\right]\right. \\
& \left.+\frac{1}{2}(\delta x(u))^{2} V^{\prime \prime}\left[x_{k_{\max }}(u)\right]+\cdots\right\rangle_{k_{\max }} .
\end{aligned}
$$

The linear term in $\delta x(u)$ vanishes when the $\{\{\mathbf{a}\}\}$ integrations are performed [as do all odd-ordered powers in $\delta x(u)]$, and the remaining terms give

$$
\begin{aligned}
\langle\Delta S\rangle_{k_{\max }}= & \frac{\beta}{2} \int_{0}^{1} d u \frac{\int d x d \mathbf{a} \exp \left[-\Sigma_{k=1}^{k_{\max }} a_{k}^{2} / 2 \sigma_{k}^{2}-\beta \bar{V}_{k_{\max }}\right] V^{\prime \prime}\left[x_{k_{\max }}(u)\right]}{\int d x d \mathbf{a} \exp \left[-\Sigma_{k=1}^{k_{\max }} a_{k}^{2} / 2 \sigma_{k}^{2}-\beta \bar{V}_{k_{\max }}\right]} \\
& \times \frac{\int d\{\{\mathbf{a}\}\} \exp \left[-\Sigma_{k=k_{\max }+1}^{\infty} a_{k}^{2} / 2 \sigma_{k}^{2}\right]\left(\sum_{k=k_{\max }}^{\infty}+a_{k} \sin k \pi u\right)^{2}}{\int d\{\{\mathbf{a}\}\} \exp \left[-\Sigma_{k=k_{\text {max }}+1}^{\infty} a_{k}^{2} / 2 \sigma_{k}^{2}\right]}+\cdots .
\end{aligned}
$$

The integration with respect to $\{\{\mathbf{a}\}\}$ in Eq. (47) has been evaluated previously in Eq. (19). Then

$$
\langle\Delta S\rangle_{k_{\max }}=\frac{\beta}{2} \int_{0}^{1} d u \frac{\int d x d \mathbf{a} \exp \left[-\Sigma_{k=1}^{k_{\max }} a_{k}^{2} / 2 \sigma_{k}^{2}-\beta \bar{V}_{k_{\max }}\right] V^{\prime \prime}\left[x_{k_{\max }}(u)\right] \sigma^{2}(u)}{\int d x d \mathbf{a} \exp \left[-\Sigma_{k=1}^{k_{\max }} a_{k}^{2} / 2 \sigma_{k}^{2}-\beta \bar{V}_{k_{\max }}\right]}+\cdots .
$$

As discussed in Sec. II C, $\sigma^{2}(u)$ decays as $1 / k_{\max }$ for large $k_{\max }$, and to leading order in $k_{\max },\langle\Delta S\rangle_{k_{\max }}$ also decays as $1 / k_{\max }$. A similar analysis of $\langle f(x) \Delta S\rangle_{k_{\max }}$ gives

$$
\langle f(x) \Delta S\rangle_{k_{\max }}=\frac{\beta}{2} \int_{0}^{1} d u \frac{\int d x d \mathbf{a} \exp \left[-\Sigma_{k=1}^{k_{\max }} a_{k}^{2} / 2 \sigma_{k}^{2}-\beta \bar{V}_{k_{\max }}\right] V^{\prime \prime}\left[x_{k_{\max }}(u)\right] f(x) \sigma^{2}(u)}{\int d x d \mathbf{a} \exp \left[-\Sigma_{k=1}^{k_{\max }} a_{k}^{2} / 2 \sigma_{k}^{2}-\beta \bar{V}_{k_{\max }}\right]}+\cdots,
$$

which also decays to zero as $1 / k_{\max }$. The leading order contribution to the error $\epsilon$ then decays as $1 / k_{\max }$ in the primitive Fourier method for operators diagonal in coordinate representation. 


\section{Path averaged properties}

Expectation values of path averaged properties like those that occur in the energy estimators require additional analysis beyond that given in Sec. III A 1. For a path averaged property $\bar{f}_{k_{\max }}$ we begin with an expression analogous to Eq. (37) in the limit that $k_{\max } \rightarrow \infty$

$$
\begin{aligned}
\left\langle\bar{f}_{\infty}\right\rangle_{\infty} & =\frac{\left\langle e^{-\Delta S} \bar{f}_{\infty}\right\rangle_{k_{\max }}}{\left\langle e^{-\Delta S}\right\rangle_{k_{\max }}} . \\
& =\left\langle\bar{f}_{\infty}\right\rangle_{k_{\max }}+\langle\Delta S\rangle_{k_{\max }}\left\langle\bar{f}_{\infty}\right\rangle_{k_{\max }}-\left\langle\Delta S \bar{f}_{\infty}\right\rangle_{k_{\max }}+\cdots .
\end{aligned}
$$

We examine the first term on the right hand side of Eq. (51) explicitly

$$
\begin{aligned}
\left\langle\bar{f}_{\infty}\right\rangle_{k_{\max }}= & \int_{0}^{1} d u\left\langle f\left[x_{k_{\max }}(u)\right]+\delta x(u) f^{\prime}\left[x_{k_{\max }}(u)\right]\right. \\
& \left.+\frac{1}{2}(\delta x(u))^{2} f^{\prime \prime}\left[x_{k_{\max }}(u)\right]+\cdots\right\rangle_{k_{\max }} \\
= & \left\langle\bar{f}_{k_{\max }}\right\rangle_{k_{\max }}+\int_{0}^{1} d u \sigma^{2}(u)\left\langle f^{\prime \prime}\left[x_{k_{\max }}(u)\right]\right\rangle_{k_{\max }}+\cdots .
\end{aligned}
$$

In obtaining Eq. (53) we have explicitly ignored the oddorder terms in $\delta x(u)$, because they vanish exactly. Then the error $\epsilon=\left\langle\bar{f}_{\infty}\right\rangle_{\infty}-\left\langle\bar{f}_{k_{\max }}\right\rangle_{k_{\max }}$ is given by

$$
\begin{aligned}
\epsilon= & \int_{0}^{1} d u \sigma^{2}(u)\left\langle f^{\prime \prime}\left[x_{k_{\max }}(u)\right]\right\rangle_{k_{\max }}+\langle\Delta S\rangle_{k_{\max }}\left\langle\bar{f}_{\infty}\right\rangle_{k_{\max }} \\
& -\left\langle\Delta S \bar{f}_{\infty}\right\rangle_{k_{\max }}+\cdots .
\end{aligned}
$$

As shown in Sec. II C the first term on the right hand side of Eq. (54) decays as $1 / k_{\max }$. By expanding $\bar{f}_{\infty}$ that appears in the fluctuation terms in Eq. (51) in a similar manner to Eq. (52), it can be shown that the leading order terms in the fluctuations in Eq. (54) also decay as $1 / k_{\max }$. Consequently, for path averaged operators, the convergence of the primitive Fourier method is $1 / k_{\max }$.

\section{B. Partial averaging}

\section{Properties diagonal in coordinate representation}

We begin this section with a discussion of the gradient partial average method, and then generalize the results to the full partial average method. We demonstrate that the asymptotic convergence rates are the same in both the gradient and full partial average methods for properties diagonal in coordinate representation.
The treatment for the errors in the gradient partial average method for a property $f(x)$ diagonal in coordinate representation follows the same development as in the primitive Fourier method. The resulting expressions for the error are easily shown to be

$$
\langle f(x)\rangle_{\infty}=\langle f(x)\rangle_{\mathrm{gpa}, k_{\max }}+\epsilon,
$$

with

$$
\begin{aligned}
& \boldsymbol{\epsilon}=\phi_{1}^{\mathrm{gpa}}+\phi_{2}^{\mathrm{gpa}}+\cdots, \\
& \phi_{1}^{\mathrm{gpa}}=\left\langle\Delta S^{\mathrm{gpa}}\right\rangle_{\mathrm{gpa}, k_{\max }}\langle f(x)\rangle_{\mathrm{gpa}, k_{\max }}-\left\langle\Delta S^{\mathrm{gpa}} f(x)\right\rangle_{\mathrm{gpa}, k_{\max }}
\end{aligned}
$$

$$
\begin{aligned}
\phi_{2}^{\mathrm{gpa}}= & \frac{1}{2}\left[\left\langle\left(\Delta S^{\mathrm{gpa}}\right)^{2} f(x)\right\rangle_{\mathrm{gpa}, k_{\max }}\right. \\
& \left.-\langle f(x)\rangle_{\mathrm{gpa}, k_{\max }}\left\langle\left(\Delta S^{\mathrm{gpa}}\right)^{2}\right\rangle_{\mathrm{gpa}, k_{\max }}\right] \\
& -\left\langle\Delta S^{\mathrm{gpa}}\right\rangle_{\mathrm{gpa}, k_{\max }}\left[\left\langle\Delta S^{\mathrm{gpa}} f(x)\right\rangle_{\mathrm{gpa}, k_{\max }}\right. \\
& \left.-\left\langle\Delta S^{\mathrm{gpa}}\right\rangle_{\mathrm{gpa}, k_{\max }}\langle f(x)\rangle_{\mathrm{gpa}, k_{\max }}\right],
\end{aligned}
$$

and so on. In Eqs. (55)-(58) we have put

$$
\begin{aligned}
& S_{k_{\max }}^{\mathrm{gpa}}(x,\{\mathbf{a}\})=\sum_{k=1}^{\infty} a_{k}^{2} / 2 \sigma_{k}^{2}+\beta \bar{V}_{\mathrm{gpa}, k_{\max }}, \\
& \langle f(x)\rangle_{\mathrm{gpa}, k_{\max }}=\frac{\int d x d\{\mathbf{a}\} \exp \left[-S_{k_{\text {max }}}^{\mathrm{gpa}}(x,\{\mathbf{a}\})\right] f(x)}{\int d x d\{\mathbf{a}\} \exp \left[-S_{k_{\max }}^{\mathrm{gpa}}(x,\{\mathbf{a}\})\right]},
\end{aligned}
$$

and

$$
\Delta S^{\mathrm{gpa}}(x,\{\mathbf{a}\})=S_{\infty}(x,\{\mathbf{a}\})-S_{k_{\max }}^{\mathrm{gpa}}(x,\{\mathbf{a}\}) .
$$

As in the primitive Fourier method we determine the convergence rate by examining the expectation value of $\Delta S^{\text {gpa }}$ with respect to the $k_{\max }$-dependent gradient partial averaged distribution

$$
\begin{aligned}
\left\langle\Delta S^{\mathrm{gpa}}\right\rangle_{\mathrm{gpa}, k_{\max }} & \beta\left\langle\bar{V}_{\infty}-\bar{V}_{\mathrm{gpa}, k_{\max }}\right\rangle_{\mathrm{gpa}, k_{\max }} \\
= & \beta \int_{0}^{1} d u\left\langle V[x(u)]-V_{\mathrm{gpa}}\left[x_{k_{\max }}(u)\right]\right\rangle_{\mathrm{gpa}, k_{\max }} \\
= & \beta \int_{0}^{1} d u\left\langle V\left[x_{k_{\max }}(u)+\delta x(u)\right]-V\left[x_{k_{\max }}(u)\right]\right. \\
& \left.-\frac{1}{2} \sigma^{2}(u) V^{\prime \prime}\left[x_{k_{\max }}(u)\right]\right\rangle_{\mathrm{gpa}, k_{\max }}
\end{aligned}
$$

As in the primitive Fourier method, we expand the first term on the right hand side of Eq. (64) in powers of $\delta x(u)$ to obtain

$$
\begin{aligned}
\left\langle\Delta S^{\mathrm{gpa}}\right\rangle_{\mathrm{gpa}, k_{\max }}= & \beta \int_{0}^{1} d u\left\langle V\left[x_{k_{\max }}(u)\right]+\delta x(u) V^{\prime}\left[x_{k_{\max }}(u)\right]+1 / 2(\delta x(u))^{2} V^{\prime \prime}\left[x_{k_{\max }}(u)\right]+1 / 3 !(\delta x(u))^{3} V^{\prime \prime \prime}\left[x_{k_{\max }}(u)\right]\right. \\
& \left.+1 / 4 !(\delta x(u))^{4} V^{\prime \prime \prime \prime}\left[x_{k_{\max }}(u)\right]+\cdots-V\left[x_{k_{\max }}(u)\right]-1 / 2 \sigma^{2}(u) V^{\prime \prime}\left[x_{k_{\max }}(u)\right]\right\rangle_{\mathrm{gpa}, k_{\max }} .
\end{aligned}
$$

The odd-order terms in $\delta x(u)$ in Eq. (65) average to zero. The quadratic term in $\delta x(u)$ on the right hand side of Eq. (65) exactly cancels the last term and the leading order term in $\left\langle\Delta S^{\mathrm{gpa}}\right\rangle_{\mathrm{gpa}, k_{\max }}$ is 


$$
\begin{aligned}
\left\langle\Delta S^{\mathrm{gpa}}\right\rangle_{\mathrm{gpa}, k_{\max }}= & \beta \int_{0}^{1} d u \frac{1}{4 !}\left\langle\left(\sum_{k=k_{\max }+1}^{\infty} a_{k} \sin k \pi u\right)^{4} V^{\prime \prime \prime \prime}\left[x_{k_{\max }}(u)\right]\right\rangle_{\mathrm{gpa}, k_{\max }} \\
= & \frac{\beta}{4 !} \int_{0}^{1} d u \frac{\int d x d \mathbf{a} \exp \left[-\sum_{k=1}^{k_{\max }} a_{k}^{2} / 2 \sigma_{k}^{2}-\beta \bar{V}_{\mathrm{gpa}, k_{\max }}\right] V^{\prime \prime \prime \prime}\left[x_{k_{\max }}(u)\right]}{\int d x d \mathbf{a} \exp \left[-\sum_{k=1}^{k_{\max }} a_{k}^{2} / 2 \sigma_{k}^{2}-\beta \bar{V}_{\left.\mathrm{gpa}, k_{\max }\right]}\right.} \\
& \times \frac{\int d\{\{\mathbf{a}\}\} \exp \left[-\sum_{k=k_{\max }+1}^{\infty} a_{k}^{2} / 2 \sigma_{k}^{2}\right]\left(\sum_{k=k_{\max }+1}^{\infty} a_{k} \sin k \pi u\right)^{4}}{\int d\{\{\mathbf{a}\}\} \exp \left[-\sum_{k=k_{\max }+1}^{\infty} a_{k}^{2} / 2 \sigma_{k}^{2}\right]}+\cdots \\
= & \frac{\beta}{192} \int_{0}^{1} d u \frac{\int d x d \mathbf{a} \exp \left[-\Sigma_{k=1}^{k_{\max }} a_{k}^{2} / 2 \sigma_{k}^{2}-\beta \bar{V}_{\mathrm{gpa}, k_{\max }}\right] V^{\prime \prime \prime \prime}\left[x_{k_{\max }}(u)\right] \sigma^{4}(u)}{\int d x d \mathbf{a} \exp \left[-\Sigma_{k=1}^{k_{\max }} a_{k}^{2} / 2 \sigma_{k}^{2}-\beta \bar{V}_{\mathrm{gpa}, k_{\max }}\right]} .
\end{aligned}
$$

Using the Euler-MacLaurin summation formula it can be shown that $\sigma^{4}(u)$ decays as $1 / k_{\max }^{2}$ as does $\left\langle\Delta S^{\mathrm{gpa}}\right\rangle_{\mathrm{gpa}, k_{\max }}$. In a similar fashion $\left\langle f(x) \Delta S^{\mathrm{gpa}}\right\rangle_{\mathrm{gpa}, k_{\max }}$ also can be shown to decay as $1 / k_{\max }^{2}$. Then in the gradient partial average method, the leading term in the error decays as $1 / k_{\max }^{2}$.

It is worth noting how the gradient partial average method improves the convergence rate of the Fourier method for coordinate diagonal properties. In the primitive Fourier method Eqs. (46)-(48) provide the leading order term in the error in inverse powers of $k_{\max }$. By seeking a modified potential that cancels the leading order terms in the error, we find an alternative route to the gradient partial average result; i.e., Eq. (23).

If the Gaussian transform of the system potential is both possible and practical, the full partial average effective potential can be used. In such cases we define

$$
S_{k_{\max }}^{\mathrm{pa}}(x,\{\mathbf{a}\})=\sum_{k=1}^{\infty} a_{k}^{2} / 2 \sigma_{k}^{2}+\beta \bar{V}_{\mathrm{pa}, k_{\max }},
$$

and

$$
\Delta S^{\mathrm{pa}}(x,\{\mathbf{a}\})=S_{\infty}(x,\{\mathbf{a}\})-S_{k_{\max }}^{\mathrm{pa}}(x,\{\mathbf{a}\}) .
$$

As in the case of gradient partial averaging, the expression for the error is given by the series of equations

$$
\langle f(x)\rangle_{\infty}=\langle f(x)\rangle_{\mathrm{pa}, k_{\max }}+\epsilon,
$$

with

$$
\begin{aligned}
\epsilon=\phi_{1}^{\mathrm{pa}} & +\phi_{2}^{\mathrm{pa}}+\cdots, \\
\phi_{1}^{\mathrm{pa}}= & \left\langle\Delta S^{\mathrm{pa}}\right\rangle_{\mathrm{pa}, k_{\max }}\langle f(x)\rangle_{\mathrm{pa}, k_{\max }}-\left\langle\Delta S^{\mathrm{pa}} f(x)\right\rangle_{\mathrm{pa}, k_{\max }}, \\
\phi_{2}^{\mathrm{pa}=} & \frac{1}{2}\left[\left\langle\left(\Delta S^{\mathrm{pa}}\right)^{2} f(x)\right\rangle_{\mathrm{pa}, k_{\max }}\right. \\
& \left.-\langle f(x)\rangle_{\mathrm{pa}, k_{\max }}\left\langle\left(\Delta S^{\mathrm{pa}}\right)^{2}\right\rangle_{\mathrm{pa}, k_{\max }}\right] \\
& -\left\langle\Delta S^{\mathrm{pa}}\right\rangle_{\mathrm{pa}, k_{\max }}\left[\left\langle\Delta S^{\mathrm{pa}} f(x)\right\rangle_{\mathrm{pa}, k_{\max }}\right. \\
& \left.-\left\langle\Delta S^{\mathrm{pa}}\right\rangle_{\mathrm{pa}, k_{\max }}\langle f(x)\rangle\right]_{\mathrm{pa}, k_{\max }}
\end{aligned}
$$

and so on. The expectation value of $\Delta S^{\mathrm{pa}}$ needed in the expression for $\phi_{1}^{\text {pa }}$ is obtained by using Eq. (22)

$$
\begin{aligned}
\left\langle\Delta S^{\mathrm{pa}}\right\rangle_{\mathrm{pa}, k_{\max }} & \\
= & \beta \int_{0}^{1} d u\left\langle V\left[x_{k_{\max }}(u)\right]+\delta x(u) V^{\prime}\left[x_{k_{\max }}(u)\right]\right. \\
& +\frac{1}{2}(\delta x(u))^{2} V^{\prime \prime}\left[x_{k_{\max }}(u)\right]+\frac{1}{3 !}(\delta x(u))^{3} V^{\prime \prime \prime}\left[x_{k_{\max }}(u)\right] \\
& +\frac{1}{4 !}(\delta x(u))^{4} V^{\prime \prime \prime \prime}\left[x_{k_{\max }}(u)\right]+\cdots-V\left[x_{k_{\max }}(u)\right] \\
& -\frac{1}{2} \sigma^{2}(u) V^{\prime \prime}\left[x_{k_{\max }}(u)\right]-\frac{1}{4 !} \sigma^{4}(u) \\
& \left.\times V^{\prime \prime \prime \prime}\left[x_{k_{\max }}(u)\right]-\cdots\right\rangle_{\mathrm{pa}, k_{\max }} .
\end{aligned}
$$

As previously the odd-order terms in $\delta x(u)$ in Eq. (75) average to zero. The terms in $\delta x(u)$ of even order $n$ average to $\sigma^{n}(u)$, so that all such even-order terms cancel exactly. Consequently, in the partial average method $\left\langle\Delta S^{\mathrm{pa}}\right\rangle_{\mathrm{pa}, k_{\max }}=0$, and the leading term in the error in the full partial average method is found in the second-order fluctuation term $\phi_{2}^{\mathrm{pa}}$.

The partial averaged effective potential introduces no special cancelations in the expression for $\left\langle\left(\Delta S^{\mathrm{pa}}\right)^{2}\right\rangle_{\mathrm{pa}, k_{\max }}$, and it is sufficient to examine the leading order decay terms for the second-order fluctuations using the primitive Fourier method. The asymptotic decay rate of the second-order fluctuations is the same in both the primitive Fourier and partial average methods. We then examine

$$
\left\langle(\Delta S)^{2}\right\rangle_{k_{\max }}=\beta^{2}\left\langle\left(\bar{V}_{\infty}-\bar{V}_{k_{\max }}\right)^{2}\right\rangle_{k_{\max }}
$$

which we expand in the usual way

$$
\begin{aligned}
\left\langle(\Delta S)^{2}\right\rangle_{k_{\max }}= & \beta^{2}\left\langle\left(\int _ { 0 } ^ { 1 } d u \left\{\delta x(u) V^{\prime}\left[x_{k_{\max }}(u)\right]\right.\right.\right. \\
& +\frac{1}{2}(\delta x(u))^{2} V^{\prime \prime}\left[x_{k_{\max }}(u)\right] \\
& +\frac{1}{3 !}(\delta x(u))^{3} V^{\prime \prime \prime}\left[x_{k_{\max }}(u)\right]
\end{aligned}
$$




$$
\begin{aligned}
& \left.\left.\left.+\frac{1}{4 !}(\delta x(u))^{4} V^{\prime \prime \prime \prime}\left[x_{k_{\max }}(u)\right]+\cdots\right\}\right)^{2}\right\rangle_{k_{\max }} \\
= & \beta^{2}\left\langle\left(\int_{0}^{1} d u \delta x(u) V^{\prime}\left[x_{k_{\max }}(u)\right]\right)^{2}\right. \\
& \left.+\left(\frac{1}{2} \int_{0}^{1} d u(\delta x(u))^{2} V^{\prime \prime}\left[x_{k_{\max }}(u)\right]\right)^{2}+\cdots\right\rangle_{k_{\max }}
\end{aligned}
$$

where in Eq. (78) we explicitly display only the two terms that we examine in detail. We begin with the first term

$$
\begin{aligned}
& T_{1}=\left\langle\left(\int_{0}^{1} d u \delta x(u) V^{\prime}\left[x_{k_{\max }}(u)\right]\right)^{2}\right\rangle_{k_{\max }} \\
& =\int d x d \mathbf{a} \exp \left[-\sum_{k=1}^{k_{\max }} a_{k}^{2} / 2 \sigma_{k}^{2}-\beta \bar{V}_{k_{\max }}\right] \\
& \times \int d\{\{\mathbf{a}\}\} \exp \left[-\sum_{k=k_{\max }+1}^{\infty} a_{k}^{2} / 2 \sigma_{k}^{2}\right] \\
& \times \int_{0}^{1} d u \int_{0}^{1} d u^{\prime} V^{\prime}\left[x_{k_{\max }}(u)\right] V^{\prime}\left[x_{k_{\max }}\left(u^{\prime}\right)\right] \\
& \times \sum_{k=k_{\max }+1}^{\infty} \sum_{k^{\prime}=k_{\max }+1}^{\infty} a_{k} a_{k^{\prime}} \sin k \pi u \sin k^{\prime} \pi u^{\prime} / \\
& \int d x d\{\mathbf{a}\} \exp \left[-\sum_{k=1}^{\infty} a_{k}^{2} / 2 \sigma_{k}^{2}-\beta \bar{V}_{k_{\max }}\right] \\
& =\int d x d \mathbf{a} \exp \left[-\sum_{k=1}^{k_{\max }} a_{k}^{2} / 2 \sigma_{k}^{2}-\beta \bar{V}_{k_{\max }}\right] \\
& \times \int d\{\{\mathbf{a}\}\} \exp \left[-\sum_{k=k_{\max }+1}^{\infty} a_{k}^{2} / 2 \sigma_{k}^{2}\right] \\
& \times \sum_{k=k_{\max }+1}^{\infty} \sum_{k^{\prime}=k_{\max }+1}^{\infty} a_{k} a_{k^{\prime}} \gamma_{k k^{\prime}}(\mathbf{a}) / \\
& \int d x d\{\mathbf{a}\} \exp \left[-\sum_{k=1}^{\infty} a_{k}^{2} / 2 \sigma_{k}^{2}-\beta \bar{V}_{k_{\max }}\right],
\end{aligned}
$$

where

$$
\begin{aligned}
\gamma_{k k^{\prime}}(\mathbf{a})= & \int_{0}^{1} d u \sin k \pi u V^{\prime}\left[x_{k_{\max }}(u)\right] \\
& \times \int_{0}^{1} d u^{\prime} \sin k^{\prime} \pi u^{\prime} V^{\prime}\left[x_{k_{\max }}\left(u^{\prime}\right)\right] .
\end{aligned}
$$

The integration with respect to $\{\{\mathbf{a}\}\}$ in Eq. (81) can be evaluated analytically to obtain

$T_{1}$

$$
=\frac{\int d x d \mathbf{a} \exp \left[-\Sigma_{k=1}^{k_{\max }} a_{k}^{2} / 2 \sigma_{k}^{2}-\beta \bar{V}_{k_{\max }}\right] \Sigma_{k=k_{\max }+1}^{\infty} \gamma_{k k}(\mathbf{a}) \sigma_{k}^{2}}{\int d x d \mathbf{a} \exp \left[-\Sigma_{k=1}^{k_{\max }} a_{k}^{2} / 2 \sigma_{k}^{2}-\beta \bar{V}_{k_{\max }}\right]} .
$$

The decay of $T_{1}$ with respect to $k_{\max }$ can be obtained from the expression

$$
\begin{aligned}
\sum_{k=k_{\max }+1}^{\infty} \gamma_{k k}(\mathbf{a}) \sigma_{k}^{2}= & \sum_{k}^{\infty} \sigma_{\max }^{\infty} \sigma_{k}^{2} \\
& \times \int_{0}^{1} d u \sin k \pi u V^{\prime}\left[x_{k_{\max }}(u)\right] \\
& \times \int_{0}^{1} d u^{\prime} \sin k \pi u^{\prime} V^{\prime}\left[x_{k_{\max }}\left(u^{\prime}\right)\right] .
\end{aligned}
$$

Each $u$-integration in Eq. (84) is recognized as the $k$ 'th Fourier sine coefficient of the expansion of $V^{\prime}\left[x_{k_{\max }}(u)\right]$ and each sine coefficient must converge asymptotically at least as fast as $1 / k$. The argument of the sum in Eq. (84) then decays as $1 / k^{4}$ so that, by the Euler-MacLaurin summation formula, $T_{1}$ itself must decay like $1 / k_{\max }^{3}$.

We next examine the second term in Eq. (78)

$$
\begin{aligned}
T_{2}= & \frac{1}{4}\left\langle\left[(\delta x(u))^{2} V^{\prime \prime}\left[x_{k_{\max }}(u)\right]\right]^{2}\right\rangle_{k_{\max }} \\
= & \frac{1}{4} \int d x d \mathbf{a} \exp \left[-\sum_{k=1}^{k_{\max }} a_{k}^{2} / 2 \sigma_{k}^{2}-\beta \bar{V}_{k_{\max }}\right] \int d\{\{\mathbf{a}\}\} \exp \left[-\sum_{k=k_{\max }+1}^{\infty} a_{k}^{2} / 2 \sigma_{k}^{2}\right] \int_{0}^{1} d u \int_{0}^{1} d u^{\prime} V^{\prime \prime}\left[x_{k_{\max }}(u)\right] V^{\prime \prime}\left[x_{k_{\max }}\left(u^{\prime}\right)\right] \\
& \times\left(\sum_{k=k_{\max }+1}^{\infty} a_{k} \sin k \pi u\right)^{2}\left(\sum_{k^{\prime}=k_{\max }+1}^{\infty} a_{k^{\prime}} \sin k^{\prime} \pi u^{\prime}\right)^{2} / \int d x d\{\mathbf{a}\} \exp \left[-\sum_{k=1}^{\infty} a_{k}^{2} / 2 \sigma_{k}^{2}-\beta \bar{V}_{k_{\max }}\right] \\
= & \frac{1}{4} d x d \mathbf{a} \exp \left[-\sum_{k=1}^{k_{\max }} a_{k}^{2} / 2 \sigma_{k}^{2}-\beta \bar{V}_{k_{\max }}\right] \sum_{k=k_{\max }+1}^{\infty} \sigma_{k}^{2} \sum_{k^{\prime}=k_{\max }+1}^{\infty} \sigma_{k^{\prime}}^{2} \\
& \times \int_{0}^{1} d u \int_{0}^{1} d u^{\prime} V^{\prime \prime}\left[x_{k_{\max }}(u)\right] V^{\prime \prime}\left[x_{k_{\max }}\left(u^{\prime}\right)\right] \sin ^{2} k \pi u \sin ^{2} k^{\prime} \pi u^{\prime} / \int d x d \mathbf{a} \exp \left[-\sum_{k=1}^{k_{\max }} a_{k}^{2} / 2 \sigma_{k}^{2}-\beta \bar{V}_{k_{\max }}\right]
\end{aligned}
$$




$$
=\frac{1}{4} \frac{\int d x d \mathbf{a} \exp \left[-\Sigma_{k=1}^{k_{\max }} a_{k}^{2} / 2 \sigma_{k}^{2}-\beta \bar{V}_{k_{\max }}\right] \int_{0}^{1} d u \int_{0}^{1} d u^{\prime} \sigma^{2}(u) \sigma^{2}\left(u^{\prime}\right) V^{\prime \prime}\left[x_{k_{\max }}(u)\right] V^{\prime \prime}\left[x_{k_{\max }}\left(u^{\prime}\right)\right]}{\int d x d \mathbf{a} \exp \left[-\sum_{k=1}^{k_{\max }} a_{k}^{2} / 2 \sigma_{k}^{2}-\beta \bar{V}_{k_{\max }}\right]} .
$$

Each factor of $\sigma^{2}(u)$ in Eq. (88) decays as $1 / k_{\max }$ so that $T_{2}$ itself decays as $1 / k_{\max }^{2}$. The remaining terms in Eq. (78) decay faster than $1 / k_{\max }^{2}$ so that $\left\langle(\Delta S)^{2}\right\rangle_{k_{\max }}$ and the error contribution from the second-order fluctuation term decay as $1 / k_{\max }^{2}$. This result implies that for properties diagonal in coordinate representation, the convergence of the property to the exact answer is $1 / k_{\max }^{2}$ with the full partial average potential used in the action. This convergence rate is identical to the gradient partial average result. In the gradient partial average method we find a modified potential that exactly cancels the leading term in the error in the primitive Fourier method in orders of inverse powers of $k_{\max }$. In the full partial average method we find a potential that exactly cancels the leading order fluctuation term in the primitive Fourier method expression for $\langle\Delta S\rangle_{k_{\max }}$; i.e., that removes the contribution of $\phi_{1}$ in Eq. (40). Both approaches lead to a result with the same convergence rate in inverse powers of $k_{\max }$.

\section{The energy}

In the primitive Fourier method the convergence rate of the energy is examined by studying the convergence rate of any path averaged operator. With partial averaging, the situation is more complex, and each specific energy estimator must be investigated separately. As is made clear in this section, we obtain $1 / k_{\max }^{2}$ convergence for the energy only if the estimator is carefully chosen.

To understand the problem, we first examine the evaluation of the average potential energy of the system. Using a theorem proved in Appendix B, the average potential energy can be evaluated using either $V(x), \bar{V}_{k_{\max }}, \bar{V}_{\mathrm{pa}, k_{\max }}$, or $\bar{V}_{\mathrm{gpa}, k_{\max }}$ as the estimator. Averages with respect to any of these four operators converge to the correct answer. However, we now show that the rates of convergence are different. In what follows the derivations are given for the gradient partial average method. The terms in Eq. (22) higher than second order in $\sigma(u)$ do not change the leading order terms in the convergence rate.

From the results of Sec. III B 1 we know the rate of convergence when $V(x)$ is used as the operator is $1 / k_{\max }^{2}$. We now analyze the error if a path averaged estimator is used as the operator instead. As in previous sections we begin the analysis with

$$
\begin{aligned}
\left\langle\bar{V}_{\infty}\right\rangle_{\infty}= & \frac{\left\langle\bar{V}_{\infty} e^{-\Delta S^{\mathrm{gpa}}}\right\rangle_{\mathrm{gpa}, k_{\max }}}{\left\langle e^{-\Delta S^{\mathrm{gpa}}}\right\rangle_{\mathrm{gpa}, k_{\max }}} \\
= & \left\langle\bar{V}_{\infty}\right\rangle_{\mathrm{gpa}, k_{\max }}+\left\langle\Delta S^{\mathrm{gpa}}\right\rangle_{\mathrm{gpa}, k_{\max }}\left\langle\bar{V}_{\infty}\right\rangle_{\mathrm{gpa}, k_{\max }} \\
& -\left\langle\Delta S^{\mathrm{gpa}} \bar{V}_{\infty}\right\rangle_{\mathrm{gpa}, k_{\max }}+\cdots .
\end{aligned}
$$

From Sec. III B 1 we know the fluctuation terms decay as $1 / k_{\max }^{2}$. We then focus on the first term on the right hand side of Eq. (90), expand it in the usual way and integrate with respect to the $\{\{\mathbf{a}\}\}$ coefficients to obtain

$$
\begin{aligned}
\left\langle\bar{V}_{\infty}\right\rangle_{\infty}= & \int_{0}^{1} d u\left\langle V\left[x_{k_{\max }}(u)\right]+\frac{1}{2} \sigma^{2}(u) V^{\prime \prime}\left[x_{k_{\max }}(u)\right]\right. \\
& +\cdots\rangle_{\mathrm{gpa}, k_{\max }}+\cdots
\end{aligned}
$$

We can now give two separate expressions for the error. The first error expression is the difference between the exact average potential energy and the average using $\bar{V}_{k_{\max }}$ as the estimator; i.e., by moving the first term on the right hand side of Eq. (91) to the left to obtain

$$
\begin{aligned}
\left\langle\bar{V}_{\infty}\right\rangle_{\infty} & -\left\langle\bar{V}_{k_{\max }}\right\rangle_{\mathrm{gpa}, k_{\max }} \\
= & \left\langle\frac{1}{2} \int_{0}^{1} d u \sigma^{2}(u) V^{\prime \prime}\left[x_{k_{\max }}(u)\right]\right\rangle_{\mathrm{gpa}, k_{\max }} \\
& +\left\langle\Delta S^{\mathrm{gpa}}\right\rangle_{\mathrm{gpa}, k_{\max }}\left\langle\bar{V}_{\infty}\right\rangle_{\mathrm{gpa}, k_{\max }}-\left\langle\Delta S^{\mathrm{gpa}} \bar{V}_{\infty}\right\rangle_{\mathrm{gpa}, k_{\max }}+\cdots
\end{aligned}
$$

Alternatively, the second error expression involves the average using $\bar{V}_{\mathrm{gpa}, k_{\max }}$ as the estimator by moving the first two terms on the right hand side of Eq. (91) to the left [see Eq. (23)] to obtain

$$
\begin{aligned}
\left\langle\bar{V}_{\infty}\right\rangle_{\infty}-\left\langle\bar{V}_{\mathrm{gpa}, k_{\max }}\right\rangle_{\mathrm{gpa}, k_{\max }} & \\
= & \left\langle\Delta S^{\mathrm{gpa}}\right\rangle_{\mathrm{gpa}, k_{\max }}\left\langle\bar{V}_{\infty}\right\rangle_{\mathrm{gpa}, k_{\max }}-\left\langle\Delta S^{\mathrm{gpa}} \bar{V}_{\infty}\right\rangle_{\mathrm{gpa}, k_{\max }}+\cdots .
\end{aligned}
$$

While the errors in both equations vanish with increasing $k_{\max }$, the decay of Eq. (92) is $1 / k_{\max }$ whereas the decay of Eq. (93) is $1 / k_{\max }^{2}$. Consequently, to obtain the optimal asymptotic convergence of the error in the potential energy using the partial average method, it is essential to use the optimal estimator.

We now examine the asymptotic convergence rates of the total energy. Because the convergence rates of the $T$-method and the virial estimator are identical, we focus on the virial estimator as well as the convergence rate of the $\mathrm{H}$-method. We begin with the virial estimator and focus on the expression in the gradient partial average method directly taken from Eq. (32) 


$$
\begin{aligned}
& \frac{1}{2}\left\langle\int_{0}^{1} \operatorname{dux}(u) V^{\prime}[x(u)]\right\rangle_{\infty} \\
& =\frac{1}{2} \int_{0}^{1} d u \frac{\left\langle x(u) V^{\prime}[x(u)] e^{-\Delta S^{\mathrm{gpa}}}\right\rangle_{\mathrm{gpa}, k_{\max }}}{\left\langle e^{-\Delta S^{\mathrm{gpa}}}\right\rangle_{\mathrm{gpa}, k_{\max }}} \\
& =\frac{1}{2} \int_{0}^{1} d u\left\langle x(u) V^{\prime}[x(u)]\right\rangle_{\mathrm{gpa}, k_{\max }} \\
& +\left\langle\Delta S^{\mathrm{gpa}}\right\rangle_{\mathrm{gpa}, k_{\max }}\left\langle\frac{1}{2} \overline{\frac{d V}{d x_{\infty}}}\right\rangle_{\mathrm{gpa}, k_{\max }} \\
& -\left\langle\Delta S^{\mathrm{gpa}} \frac{1}{2} x \frac{d V}{d x_{\infty}}\right\rangle_{\mathrm{gpa}, k_{\max }}+\cdots .
\end{aligned}
$$

From Sec. III B 1 we know the fluctuation terms vanish like $1 / k_{\max }^{2}$, and we concentrate on the first term on the right hand side of Eq. (95). Then expanding in the usual way

$$
\begin{aligned}
& \frac{1}{2}\left\langle\left.\int_{0}^{1} d u x(u) V^{\prime}[x(u)]\right|_{\infty}\right. \\
& =\frac{1}{2} \int_{0}^{1} d u\left\langle[ x _ { k _ { \operatorname { m a x } } } ( u ) + \delta x ( u ) ] \left[ V^{\prime}\left[x_{k_{\max }}(u)\right]\right.\right. \\
& \quad+\delta x(u) V^{\prime \prime}\left[x_{k_{\max }}(u)\right]+1 / 2(\delta x(u))^{2} V^{\prime \prime \prime}\left[x_{k_{\max }}(u)\right] \\
& \quad+\cdots]\rangle_{\mathrm{gpa}, k_{\max }}+\cdots .
\end{aligned}
$$

We next combine terms to the same order in $\delta x(u)$ and analytically evaluate the integrals with respect to $\{\{\mathbf{a}\}\}$ to obtain

$$
\begin{aligned}
& \frac{1}{2}\left\langle\int_{0}^{1} d u x(u) V^{\prime}[x(u)]\right\rangle_{\infty} \\
& =\frac{1}{2} \int_{0}^{1} d u\left\{\left\langle x_{k_{\max }}(u) V^{\prime}\left[x_{k_{\max }}(u)\right]\right\rangle_{\mathrm{gpa}, k_{\max }}\right. \\
& \quad+\frac{1}{2} \sigma^{2}(u)\left\langle x_{k_{\max }}(u) V^{\prime \prime \prime}\left[x_{k_{\max }}(u)\right]\right\rangle_{\mathrm{gpa}, k_{\max }} \\
& \left.\quad+\sigma^{2}(u)\left\langle V^{\prime \prime}\left[x_{k_{\max }}(u)\right]\right\rangle_{\mathrm{gpa}, k_{\max }}\right\}+\cdots .
\end{aligned}
$$

The first two terms on the right hand side of Eq. (97) combine to produce the gradient partial average expression for the first term of the virial estimator. Moving the result to the left hand side of Eq. (97) we obtain

$$
\begin{aligned}
& \frac{1}{2}\left\langle\overline{x(u) V^{\prime}[x(u)]_{\infty}}\right\rangle_{\infty}-\frac{1}{2}\left\langle\overline{\left.x(u) V^{\prime}[x(u)]_{\mathrm{gpa}, k_{\max }}\right\rangle_{\mathrm{gpa}, k_{\max }}}\right. \\
& =\int_{0}^{1} d u\left\langle\frac{1}{2} \sigma^{2}(u) V^{\prime \prime}\left[x_{k_{\max }}(u)\right]\right\rangle_{\mathrm{gpa}, k_{\max }} \\
& \quad+\mathcal{O}\left(1 / k_{\max }^{2}\right)
\end{aligned}
$$

where $\mathcal{O}\left(1 / k_{\max }^{2}\right)$ represents the terms that decay like $1 / k_{\max }^{2}$ that have not been displayed explicitly in Eq. (98). The first term on the right hand side of Eq. (98) decays like $1 / k_{\max }$. However, from Eq. (23) we know that

$$
V_{\mathrm{gpa}}\left[x_{k_{\max }}(u)\right]-V\left[x_{k_{\max }}(u)\right]=\frac{1}{2} \sigma^{2}(u) V^{\prime \prime}\left[x_{k_{\max }}(u)\right] .
$$

We can then combine Eqs. (98) and (99) to obtain the expression for the error when the virial estimator is used to calculate the total energy

$$
\begin{aligned}
& \left\langle\frac{1}{2}{\overline{x(u) V^{\prime}[x(u)]_{\infty}}}_{\infty}+\bar{V}_{\infty}\right\rangle_{\infty}-\left\langle\frac{1}{2}{\overline{x(u) V^{\prime}[x(u)]_{\mathrm{gpa}, k_{\text {max }}}}}\right. \\
& \left.+2 \bar{V}_{\mathrm{gpa}, k_{\max }}-\bar{V}_{k_{\max }}\right\rangle_{k_{\max }}=\mathcal{O}\left(1 / k_{\max }^{2}\right) .
\end{aligned}
$$

The $1 / k_{\max }^{2}$ decay in the error using the virial estimator is found only for the estimator given in Eq. (32). Related estimators like $\left\langle\frac{1}{2} \overline{x(u) V_{\mathrm{gpa}, k_{\max }[x(u)]}^{\prime}}+\bar{V}_{k_{\max }}\right\rangle$ converge to the correct result but only as $1 / k_{\max }$. If the full partial average potential rather than the gradient partial average potential is used in the analysis, the result is identical.

We next consider the asymptotic rate of convergence of the energy when the $H$-estimator is used. The first term on the right hand side of Eq. (25) is the point potential energy estimator, and the error in the potential energy decays like $1 / k_{\max }^{2}$ from the discussion given in Sec. III B 1 . Because the second term on the right hand side of Eq. (25) is a constant, we need examine the decay of the errors in the last two terms on the right hand side of Eq. (25). We begin with the last term and again limit the discussion to the gradient partial average method. The analysis using the full partial average method is the same, because the terms beyond second-order in $\sigma(u)$ in Eq. (22) do not contribute to the leading order terms in the convergence rate. We write

$$
\begin{aligned}
& \left\langle\int_{0}^{1} d u(1-u)^{2} V^{\prime \prime}[x(u)]\right\rangle_{\infty} \\
& \quad=\int_{0}^{1} d u \frac{\left\langle(1-u)^{2} V^{\prime \prime}[x(u)] e^{-\Delta S^{\mathrm{gpa}}}\right\rangle_{\mathrm{gpa}, k_{\max }}}{\left\langle e^{-\Delta S^{\mathrm{gpa}}}\right\rangle_{\mathrm{gpa}, k_{\max }}} \\
& =\int_{0}^{1} d u(1-u)^{2}\left\langle V^{\prime \prime}[x(u)]\right\rangle_{\mathrm{gpa}, k_{\max }}+\mathcal{O}\left(1 / k_{\max }^{2}\right),
\end{aligned}
$$

where $\mathcal{O}\left(1 / k_{\max }^{2}\right)$ represents the fluctuation terms that we already have shown converge like $1 / k_{\max }^{2}$. We next expand Eq. (102) in the usual way followed by an integration with respect to the $\{\{\mathbf{a}\}\}$ variables to obtain

$$
\begin{aligned}
\left\langle\int_{0}^{1} d u(1-u)^{2} V^{\prime \prime}[x(u)]\right\rangle_{\infty} \\
=\int_{0}^{1} d u(1-u)^{2}\left\langle V^{\prime \prime}\left[x_{k_{\max }}(u)\right]+\delta x(u) V^{\prime \prime \prime}\left[x_{k_{\max }}(u)\right]\right. \\
\left.\quad+\frac{1}{2}(\delta x(u))^{2} V^{\prime \prime \prime \prime}\left[x_{k_{\max }}(u)\right]+\cdots\right\rangle_{\mathrm{gpa}, k_{\max }}+\mathcal{O}\left(1 / k_{\max }^{2}\right) \\
=\int_{0}^{1} d u(1-u)^{2}\left\langle V^{\prime \prime}\left[x_{k_{\max }}(u)\right]+\frac{1}{2} \sigma^{2}(u) V^{\prime \prime \prime \prime}\left[x_{k_{\max }}(u)\right]\right. \\
\quad+\cdots\rangle_{\mathrm{gpa}, k_{\max }}+\mathcal{O}\left(1 / k_{\max }^{2}\right) .
\end{aligned}
$$


The first two terms in the average on the right hand side of Eq. (104) combine to give the gradient partial average potential, and we obtain

$$
\begin{aligned}
& \left\langle\int_{0}^{1} d u(1-u)^{2} V^{\prime \prime}[x(u)]\right\rangle_{\infty} \\
& \quad-\left\langle\int_{0}^{1} d u(1-u)^{2} V_{\mathrm{gpa}}^{\prime \prime}\left[x_{k_{\max }}(u)\right]\right\rangle_{\mathrm{gpa}, k_{\max }}=\mathcal{O}\left(1 / k_{\max }^{2}\right) .
\end{aligned}
$$

The error in the third term on the right hand side of Eq. (25) can be obtained from

$$
\begin{aligned}
& \left\langle\left(\int_{0}^{1} d u(1-u) V^{\prime}[x(u)]\right)^{2}\right\rangle_{\infty} \\
& =\frac{\left\langle\left(\int_{0}^{1} d u(1-u) V^{\prime}[x(u)]\right)^{2} e^{-\Delta S^{\mathrm{gpa}}}\right\rangle_{\mathrm{gpa}, k_{\max }}}{\left\langle e^{-\Delta S^{\mathrm{gpa}}}\right\rangle_{\mathrm{gpa}, k_{\max }}} \\
& =\left\langle\left(\int_{0}^{1} d u(1-u) V^{\prime}[x(u)]\right)^{2}\right\rangle_{\mathrm{gpa}, k_{\max }}+\cdots,
\end{aligned}
$$

where the terms not explicitly displayed in Eq. (107) are fluctuation terms and decay at least as rapidly as $1 / k_{\max }^{2}$. Expanding Eq. (107) in the usual way, we obtain

$$
\begin{aligned}
\left\langle\left(\int_{0}^{1} d u(1-u) V^{\prime}[x(u)]\right)^{2}\right\rangle_{\infty}= & \left\langle\int _ { 0 } ^ { 1 } d u \int _ { 0 } ^ { 1 } d u ^ { \prime } ( 1 - u ) ( 1 - u ^ { \prime } ) \left\{ V^{\prime}\left[x_{k_{\max }}(u)\right]+\delta x(u) V^{\prime \prime}\left[x_{k_{\max }}(u)\right]\right.\right. \\
& \left.+\frac{1}{2}(\delta x(u))^{2} V^{\prime \prime \prime}\left[x_{k_{\max }}(u)\right]+\cdots\right\}\left\{V^{\prime}\left[x_{k_{\max }}\left(u^{\prime}\right)\right]+\delta x\left(u^{\prime}\right) V^{\prime \prime}\left[x_{k_{\max }}\left(u^{\prime}\right)\right]\right. \\
& \left.\left.+\frac{1}{2}\left(\delta x\left(u^{\prime}\right)\right)^{2} V^{\prime \prime \prime}\left[x_{k_{\max }}\left(u^{\prime}\right)\right]+\cdots\right\}\right\rangle_{\mathrm{gpa}, k_{\max }}+\cdots
\end{aligned}
$$

We next combine like powers in $\delta x(u)$ and integrate analytically with respect to $\{\{\mathbf{a}\}\}$ to obtain

$$
\begin{aligned}
\left\langle\left(\int_{0}^{1} d u(1-u) V^{\prime}[x(u)]\right)^{2}\right\rangle_{\infty} \\
=\left\langle\int_{0}^{1} d u(1-u) \int_{0}^{1} d u^{\prime}\left(1-u^{\prime}\right)\right. \\
\quad \times\left\{V^{\prime}\left[x_{k_{\max }}(u)\right]+\frac{1}{2} \sigma^{2}(u) V^{\prime \prime \prime}\left[x_{k_{\max }}(u)\right]\right\} \\
\left.\quad \times\left\{V^{\prime}\left[x_{k_{\max }}\left(u^{\prime}\right)\right]+\frac{1}{2} \sigma^{2}\left(u^{\prime}\right) V^{\prime \prime \prime}\left[x_{k_{\max }}\left(u^{\prime}\right)\right]\right\}\right\rangle_{\mathrm{gpa}, k_{\max }} \\
\quad+\left\langle\sum_{k=k_{\max }+1}^{\infty} \Gamma_{k k}(\mathbf{a}) \sigma_{k}^{2}\right\rangle_{\mathrm{gpa}, k_{\max }}+\cdots,
\end{aligned}
$$

where

$$
\begin{aligned}
\Gamma_{k k^{\prime}}(\mathbf{a})= & \int_{0}^{1} d u(1-u) \sin k \pi u V^{\prime \prime}\left[x_{k_{\max }}(u)\right] \\
& \times \int_{0}^{1} d u^{\prime}\left(1-u^{\prime}\right) \sin k^{\prime} \pi u^{\prime} V^{\prime \prime}\left[x_{k_{\max }}\left(u^{\prime}\right)\right] .
\end{aligned}
$$

As discussed in reference to Eq. (84), each factor in the definition of $\Gamma_{k k^{\prime}}(\mathbf{a})$ is the Fourier sine coefficient of (1 $-u) V^{\prime \prime}\left[x_{k_{\max }}(u)\right]$ and decays asymptotically at least as fast as $1 / k$. Consequently, the last term displayed on the right hand side of Eq. (109) decays at least as fast as $1 / k_{\max }^{3}$ and the error term

$$
\begin{aligned}
& \left\langle\left(\int_{0}^{1} d u(1-u) V^{\prime}[x(u)]\right)^{2}\right\rangle_{\infty} \\
& \quad-\left\langle\left(\int_{0}^{1} d u(1-u) V_{\mathrm{gpa}}^{\prime}[x(u)]\right)^{2}\right\rangle_{\mathrm{gpa}, k_{\max }},
\end{aligned}
$$

decays at least as fast as $1 / k_{\max }^{2}$. Finally, we conclude the error in the $H$-method estimate of the energy also converges as $1 / k_{\max }^{2}$.

\section{NUMERICAL EXAMPLES}

In this section we illustrate the results of the derivations in Sec. III with some example calculations on onedimensional systems. We choose to examine onedimensional systems, because for such simple systems it is possible to determine the exact result for comparisons, and it is possible to include many Monte Carlo points to insure good statistics.

The first system is the double-well potential used and described previously to demonstrate the j-walking method. ${ }^{19}$ We choose the potential parameters so that the higher energy well is $90 \%$ the depth of the lower energy well. Because this potential is a polynomial through fourth-order, exact results are obtained by matrix diagonalization in a harmonic oscillator basis set. The system mass is taken to be $1 \%$ the mass of a proton. With this mass the system has two sets of nearly degenerate eigenvalues below the top of the barrier that separates the two potential minima. The temperature is taken to be $\beta \Delta E_{01}=5$ where $\Delta E_{01}$ is the energy separating the ground and first excited state of the system. Figure 1 is a plot of the total energy of this system as determined with the 

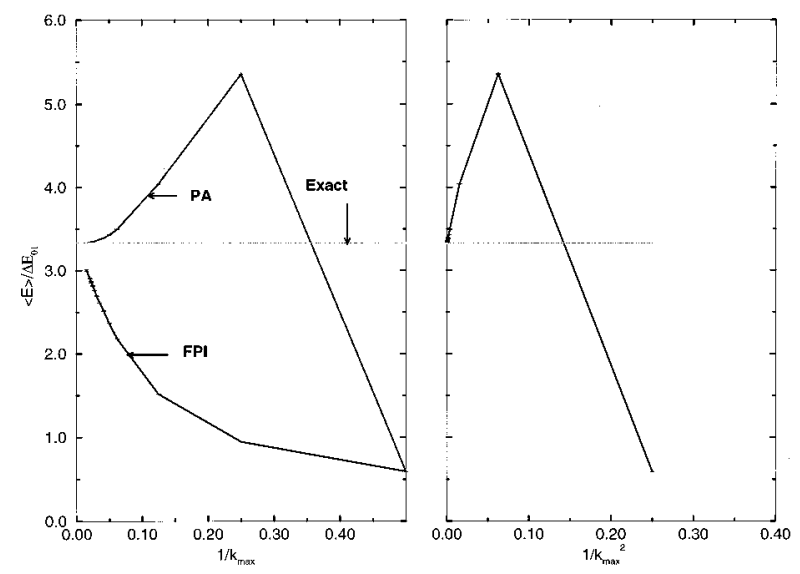

FIG. 1. The left panel compares the total energy of the double-well potential as a function of $1 / k_{\max }$ determined using the virial estimator. The line labeled FPI represents the primitive Fourier method and the line labeled PA represents the results with partial averaging. The linear approach of the primitive Fourier results to the exact energy is characteristic of $1 / k_{\max }$ convergence, and the approach with zero slope of the partial averaged results to the exact energy is characteristic of convergence that is more rapid than $1 / k_{\max }$. In the right panel the partial averaged results are plotted as a function of $1 / k_{\max }^{2}$, and the linear behavior provides numerical verification of the asymptotic $1 / k_{\max }^{2}$ convergence rates of the partial average method. The error bars displayed in this and subsequent figures represent one standard deviation of the mean.

virial estimator [Eq. (32)]. In the left hand panel both the primitive Fourier path integral method (labeled FPI) and the partial averaged results (labeled PA) are displayed as a function of $1 / k_{\max }$. The primitive Fourier results approach the exact energy (represented by the horizontal line) linearly. The partial average results approach the exact result with a vanishing slope. Such behavior is consistent with the asymptotic convergence rate expected from the analysis. In the right hand panel, the partial average results are plotted as a function of $1 / k_{\max }^{2}$, and the linear approach to the correct result is consistent with the expected asymptotic convergence of the virial estimator with partial averaging.

A second example of the asymptotic convergence characteristics of the total energy is displayed in Fig. 2. The potential energy for the model problem is given by

$$
V(x)=V_{\mathrm{LJ}}(x+\alpha)+V_{\mathrm{LJ}}(x-\alpha)
$$

where $V_{\mathrm{LJ}}(x)$ is the standard Lennard-Jones interaction

$$
V_{\mathrm{LJ}}(x)=4 \epsilon\left[\left(\frac{\sigma}{x}\right)^{12}-\left(\frac{\sigma}{x}\right)^{6}\right]
$$

and $\alpha$ is taken to be $1.2 \sigma$. The parameters $\epsilon$ and $\sigma$ are, respectively, the standard Lennard-Jones energy and length parameters. This potential has been used previously as a onedimensional representation for a fluid. ${ }^{20}$ The Lennard-Jones parameters and mass have been taken from a recent study by Chakravarty, Gordillo and Ceperley ${ }^{21}$ on clusters of hydrogen molecules. In Fig. 2 we display the average energy in units of $\epsilon$ determined using the virial estimator as a function

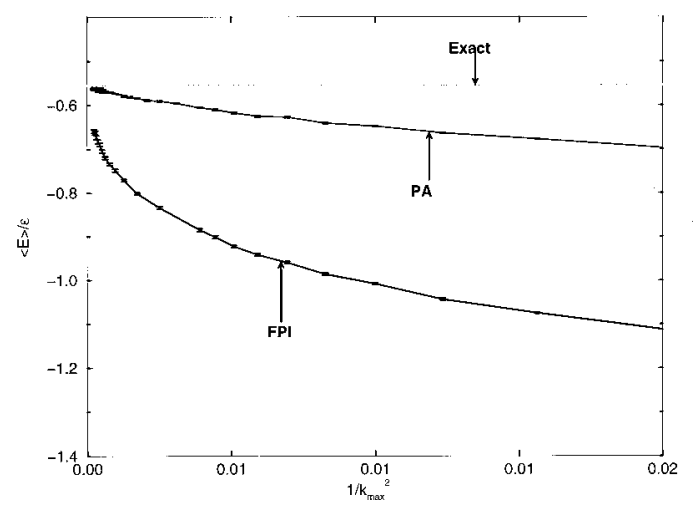

FIG. 2. The average energy of the Lennard-Jones cage potential discussed in the text as a function of $1 / k_{\max }^{2}$. The energy has been determined using the virial estimator. The linear convergence of the partial averaged results (labeled PA on the graph) with $1 / k_{\max }^{2}$ and the less rapid convergence of the primitive Fourier method results (labeled FPI) on the graph are evident. The exact results have been obtained using numerical matrix multiplication methods.

of $1 / k_{\max }^{2}$ both with and without partial averaging. The linear convergence of the partial averaged results with $1 / k_{\max }^{2}$ is evident, as is the less rapid convergence of the primitive Fourier method.

The data in Fig. 1 do not converge to the exact result monotonically prior to the asymptotic region. In some cases such nonmonotonic convergence can lead to incorrect conclusions about the ultimate convergence of a calculation. The analysis of this work can help prevent such incorrect conclusions. To illustrate this point, in Fig. 3 we plot the average

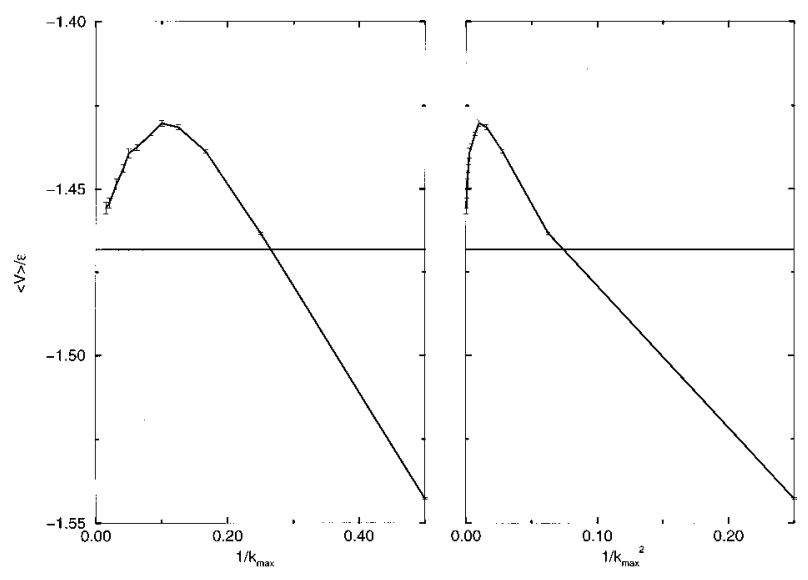

FIG. 3. The average potential energy as a function of $1 / k_{\max }$ (left panel) and $1 / k_{\max }^{2}$ (right panel) for the quartic oscillator using the primitive Fourier method. As discussed in the text, the parameters for the quartic potential are chosen to fit the potential discussed in Fig. 2, and the average potential energy is given in units of the $\epsilon$ parameter used in the Lennard-Jones cage potential. The asymptotic linear convergence to the exact result is evident. The plateau near $k_{\max }=10$ provides an example of false convergence, because the convergence is not linear in $1 / k_{\max }$. Such behavior is evidence that more Fourier coefficients are needed in a calculation. The convergence appears to be linear in $1 / k_{\max }^{2}$ for small $k_{\max }$, but becomes linear in $1 / k_{\max }$ only in the asymptotic region. The horizontal line in each panel represents the exact result. 
potential energy of a quantum simulation using a onedimensional quartic potential of the form

$$
V(x)=a_{0}+a_{4} x^{4},
$$

as a function of $1 / k_{\max }$ determined with the primitive Fourier method. The coefficients $a_{0}$ and $a_{4}$ are chosen to fit the potential function given in Eq. (111). From the analysis presented in this work, we know the convergence is linear in the asymptotic region, and the linear approach to the exact result is evident for large $k_{\max }$. However, at intermediate values of $k_{\max }$ near $k_{\max }=8-16$, the average potential energy changes little and appears to be converged. In practice, if the exact result is not known, truncation of the calculation at some value of $k_{\max }$ between 8 and 16 appears to be natural. However, in the region of intermediate values of $k_{\max }$ the asymptotic behavior is not $1 / k_{\max }$ as is required by the analysis of this work. Consequently, we have clear evidence the calculation is not converged prior to including more Fourier coefficients in the calculation.

\section{DISCUSSION}

There is the case of one physical system where the convergence rates for calculated thermodynamic properties are more rapid than the rates derived in this work. For the onedimensional harmonic oscillator partial averaging introduces a modification to the density matrix that is dependent on temperature but independent of coordinates. ${ }^{4}$ Many expectation values derived from the partial averaged density matrix for the one-dimensional oscillator are identical to those derived from the primitive Fourier method. Additionally, expectation values of coordinate dependent properties for the one-dimensional oscillator converge as $1 / k_{\max }^{3}$ in the primitive Fourier method. ${ }^{4}$ This increased convergence can be understood by noting that the second derivative of the potential energy with respect to its coordinate is a constant, and the first fluctuation term defined in Eq. (41) vanishes. The leading contributions to the second fluctuation term defined in Eq. (42) can be shown to converge as $1 / k_{\max }^{3}$ for the oscillator. While the oscillator is an uncommon example of a system whose properties converge more rapidly than others with respect to the number of Fourier coefficients included, we include the qualification "at least" to the following statements that summarize our findings:

1. The errors in the expectation values of all properties calculated using the primitive Fourier method converge asymptotically at least as rapidly as $1 / k_{\max }$.

2. The errors in the expectation values of all properties diagonal in coordinate representation using either the full partial average method or the gradient partial average method converge asymptotically at least as rapidly as $1 / k_{\max }^{2}$.
3. The errors in the expectation values of any of the energy estimators developed in Sec. II D using either the full partial average method or the gradient partial average method converge asymptotically at least as rapidly as $1 / k_{\max }^{2}$. Alternate estimators of the energy can converge less rapidly than $1 / k_{\max }^{2}$, so that the choice of optimal estimator is important to achieve optimal convergence.

In addition to the results outlined above the formal methods have provided new insight into the partial average method. A formal demonstration has been given that the gradient partial average and the full partial average methods provide identical levels of enhancement of the asymptotic convergence rates. Furthermore, we have made clear that the gradient partial average method works by introducing exact cancelation of the leading terms in the error incurred by inclusion of a finite set of Fourier coefficients.

With analytic asymptotic convergence rates, a signature is available that indicates when a calculation has converged with respect to the number of Fourier coefficients included. Erroneous truncations of path integral simulations that arise from nonmonotonic convergence characteristics can be avoided. Furthermore, once it has been determined that the number of Fourier coefficients included is in the asymptotic region, extrapolation methods to the exact result can be introduced with some confidence.

In the derivations it is assumed that averages of increasing powers of $\Delta S$ decay with increasing powers of $1 / k_{\max }$. While we have no formal proof of this assertion, detailed examination has always proved the assumption to be valid.

\section{ACKNOWLEDGMENTS}

This work has been supported in part by the National Science Foundation under grant Nos. CHE-9411000, CHE9714970, and CHE-9625498.

\section{APPENDIX A: THE N-PARTICLE VIRIAL ESTIMATOR}

In this appendix we show that for $N$-particle systems if the potential energy is independent of the coordinate of the center of mass, the virial estimator results in an energy from the internal coordinates only; i.e., an energy that does not include the constant contribution from the motion of the center of mass. It is easiest to understand this result in terms of the classical virial first. A derivation is then sketched for the quantum virial. We limit the discussion to $N$ particles in one dimension, and assume all particles have the same mass. The extension to three dimensions with particles of mixed mass is straight forward.

The expression for the classical virial is

$$
\begin{aligned}
\alpha & =\left\langle\sum_{i=1}^{N} x_{i} \frac{\partial}{\partial x_{i}} V\right\rangle \\
& =\frac{\int d x_{1} d x_{2} \cdots d x_{N} \Sigma_{i=1}^{N} x_{i}\left(\partial / \partial x_{i}\right) V\left(x_{1}, x_{2}, \ldots, x_{N}\right) \exp \left[-\beta V\left(x_{1}, x_{2}, \ldots, x_{N}\right)\right]}{\int d x_{1} d x_{2} \cdots d x_{N} \exp \left[-\beta V\left(x_{1}, x_{2}, \ldots, x_{N}\right)\right]} .
\end{aligned}
$$


We next introduce the coordinate of the center of mass

$$
x=\frac{1}{N} \sum_{i=1}^{N} x_{i}
$$

and the relative coordinates

$$
x_{i}^{\prime}=x_{i}-x
$$

$$
=\frac{1}{N}\left[(N-1) x_{i}-\sum_{j \neq i}^{N} x_{j}\right] \text {. }
$$

On substitution of the center of mass and relative coordinates in Eq. (A2) we obtain

$$
\begin{aligned}
\alpha & =\left\langle\left\{\sum_{i=1}^{N-1} \frac{x_{i}^{\prime}+x}{N}\left[(N-1) \frac{\partial}{\partial x_{i}^{\prime}}-\sum_{j \neq i}^{N-1} \frac{\partial}{\partial x_{j}^{\prime}}+\frac{\partial}{\partial x}\right]+\frac{x-\sum_{j=1}^{N-1} x_{j}^{\prime}}{N}\left[-\sum_{j=1}^{N-1} \frac{\partial}{\partial x_{j}^{\prime}}+\frac{\partial}{\partial x}\right]\right\} V\right\rangle \\
& =\frac{\int d x_{1}^{\prime} d x_{2}^{\prime} \cdots d x_{N-1}^{\prime} d x \sum_{i=1}^{N-1} x_{i}^{\prime}\left(\partial / \partial x_{i}^{\prime}\right) V\left(x_{1}^{\prime}, x_{2}^{\prime}, \ldots, x_{N-1}^{\prime}\right) \exp \left[-\beta V\left(x_{1}^{\prime}, x_{2}^{\prime}, \ldots, x_{N-1}^{\prime}\right)\right]}{\int d x_{1}^{\prime} d x_{2}^{\prime} \cdots d x_{N-1}^{\prime} d x \exp \left[-\beta V\left(x_{1}^{\prime}, x_{2}^{\prime}, \ldots, x_{N-1}^{\prime}\right)\right]},
\end{aligned}
$$

where we have explicitly used the independence of the potential energy on the coordinate $x$. The contributions from the $x$-integration cancel in the numerator and the denominator in Eq. (A7). After integration by parts and assuming the surface terms vanish, we obtain the final result

$$
\frac{1}{2}\left\langle\sum_{i=1}^{N} x_{i} \frac{\partial V}{\partial x_{i}}\right\rangle=\frac{N-1}{2 \beta},
$$

which is the total kinetic energy less the kinetic energy of the center of mass of the system. This completes the proof of the classical virial result.

The proof of the quantum virial result proceeds in a similar fashion to the classical derivation. We begin with the quantum expression using Fourier path integral partial average methods with $k_{\max }$ Fourier coefficients included

$$
\begin{aligned}
\alpha= & \left\langle\left(\sum_{i=1}^{N} x_{i} \frac{\partial}{\partial x_{i}}+\sum_{i=1}^{N} \sum_{k=1}^{k_{\max }} a_{k, i} \frac{\partial}{\partial a_{k, i}}\right)\right. \\
& \left.\times\left(\sum_{i=1}^{N} \sum_{k=1}^{k_{\max }} a_{k, i}^{2} / 2 \sigma_{k}^{2}+\beta \bar{V}_{\mathrm{pa}, k_{\max }}\right)\right\rangle_{k_{\max }} .
\end{aligned}
$$

In Eq. (A9) we have included the required $N k_{\max }$ Fourier coefficients labeled by Fourier index $k$ and particle index $i$, and for simplicity we have assumed all particle have the same mass. The center of mass transformation in the quantum case applies to the entire path so that we write

$$
x_{\mathrm{cm}, k_{\max }}(u)=\frac{1}{N} \sum_{i=1}^{N} x_{i, k_{\max }}(u),
$$

where

$$
x_{i, k_{\max }}(u)=x_{i}+\sum_{k=1}^{k_{\max }} a_{k, i} \sin k \pi u .
$$

We then define the relative coordinates for the entire path

$$
\begin{aligned}
x_{i, k_{\max }}^{\prime}(u) & =x_{i, k_{\max }}(u)-x_{\mathrm{cm}, k_{\max }}(u) \\
& =x_{i}^{\prime}+\sum_{k=1}^{k_{\max }} a_{k, i}^{\prime} \sin k \pi u,
\end{aligned}
$$

where $x_{i}^{\prime}$ is defined as in Eq. (A4), and

$$
a_{k, i}^{\prime}=a_{k, i}-A_{k},
$$

with

$$
A_{k}=\frac{1}{N} \sum_{i=1}^{N} a_{k, i} .
$$

The center of mass transformation is then introduced into Eq. (A9) resulting in the expression

$$
\begin{aligned}
\alpha= & \left\langle\left\{\sum_{i=1}^{N-1} \frac{x_{i}^{\prime}+x}{N}\left[(N-1) \frac{\partial}{\partial x_{i}^{\prime}}-\sum_{j \neq i}^{N} \frac{\partial}{\partial x_{j}^{\prime}}+\frac{\partial}{\partial x}\right]+\frac{x-\sum_{j=1}^{N-1} x_{j}^{\prime}}{N}\left[-\sum_{j=1}^{N-1} \frac{\partial}{\partial x_{j}^{\prime}}+\frac{\partial}{\partial x}\right]\right.\right. \\
& \left.+\sum_{k=1}^{k_{\max }} \sum_{i=1}^{N-1} \frac{a_{k, i}^{\prime}+A_{k}}{N}\left[(N-1) \frac{\partial}{\partial a_{k, i}^{\prime}}-\sum_{j \neq i}^{N-1} \frac{\partial}{\partial a_{k, j}^{\prime}}+\frac{\partial}{\partial A_{k}}\right]+\sum_{k=1}^{k_{\max }} \frac{A_{k}-\sum_{j=1}^{N-1} a_{k, j}^{\prime}}{N}\left[-\sum_{j=1}^{N-1} \frac{\partial}{\partial a_{k, j}^{\prime}}+\frac{\partial}{\partial A_{k}}\right]\right\} \\
& \left.\times\left\{\sum_{i=1}^{N-1} \sum_{k=1}^{k_{\max }} \frac{\left(a_{k, i}^{\prime}+A_{k}\right)^{2}}{2 \sigma_{k}^{2}}+\sum_{k=1}^{k_{\max }} \frac{\left(A_{k}-\sum_{j=1}^{N-1} a_{k, j}^{\prime}\right)^{2}}{2 \sigma_{k}^{2}}+\int_{0}^{1} d u V_{\mathrm{pa}}\left[x_{1, k_{\max }^{\prime}}(u), x_{2, k_{\max }}(u), \ldots, x_{N-1, k_{\max }}^{\prime}(u)\right]\right\}\right)
\end{aligned}
$$


Equation (A16) can be integrated by parts to give

$$
\alpha=N k_{\text {max }}+N-1,
$$

where the surface terms are assumed to vanish, and the independence of the potential energy $V$ on the center of mass coordinate $x$ is used explicitly. Using the $T$-method expression for the total energy for an $N$-particle system in one spatial dimension

$$
\begin{aligned}
\langle E\rangle= & \frac{N\left(k_{\max }+1\right)}{2 \beta}+\left\langle 2 \bar{V}_{\mathrm{pa}, k_{\max }}-\bar{V}_{k_{\max }}\right. \\
& \left.-\sum_{k=1}^{k_{\max }} \sum_{i=1}^{N} a_{k, i}^{2} / 2 \beta \sigma_{k}^{2}\right\rangle_{k_{\max }},
\end{aligned}
$$

Eqs. (A17) and (A18) can be combined to give

$$
\begin{aligned}
\langle E\rangle= & \left\langle\frac{1}{2} \sum_{i=1}^{N} \frac{x_{i, k_{\max }}(u) V_{\mathrm{pa}, k \max }^{\prime}\left[x_{i, k_{\max }}(u)\right]}{{ }}\right. \\
& \left.+2 \bar{V}_{\mathrm{pa}, k_{\max }}-\bar{V}_{k_{\max }}\right\rangle_{k_{\max }}+\frac{1}{2 \beta},
\end{aligned}
$$

i.e., for systems whose potential energy is independent of the coordinate of the center of mass, the virial estimator gives the total energy less the energy of the free translational motion of the center of mass.

\section{APPENDIX B: THE EQUIVALENCE OF POINT AND PATH ESTIMATORS}

In Sec. III B 2 we discussed alternate estimators of the potential energy that all converge to the correct result with an infinite set of Fourier coefficients, but with different rates of convergence. Here we prove a theorem and a lemma that justifies that all the estimators must converge to the same result.

Theorem: Let $x(u)$ represent a path for $0 \leqslant u \leqslant \beta \hbar$ and let $x(t)$ represent some point on the path. Let $f(x)$ be a function of $x$ and write the expectation value of $f(x)$ at point $t$ by

$$
\langle f[x(t)]\rangle=\int d x \int_{x}^{x} D x(u) e^{-S[x(u)] / \hbar} f[x(t)],
$$

where $S[x(u)]$ is the action associated with path $x(u)$ and the path integral is taken over all paths that start and end at point $x$. Then

$$
\langle f[x(t)]\rangle=\langle f(x)\rangle,
$$

i.e., the expectation value is independent of the time point $t$.

Proof: We write

$$
\int_{x}^{x} D x(u) e^{-S[x(u)] / \hbar}=\int d x(t) \int_{x(t)}^{x(t)} D x(u) e^{-S[x(u)] / \hbar},
$$

where the notation $\int_{x(t)}^{x(t)} D x(u)$ implies a sum over all paths that are constrained to pass through the point $x(t)$ at time $t$. We can then write

$$
\begin{aligned}
\langle f[x(t)]\rangle & \\
= & \int d x \int d x(t) \int_{x(t)}^{x(\beta \hbar)} D x(u) e^{-S[x(u)] / \hbar} f[x(t)] \\
& \times \int_{x(0)}^{x(t)} D x(u) e^{-S[x(u)] / \hbar} \\
= & \int d x \int d x(t)\left\langle x\left|e^{-(\beta \hbar-t) \hat{H} / \hbar} f[x(t)]\right| x(t)\right\rangle \\
& \times\left\langle x(t)\left|e^{-\hat{H} t / \hbar}\right| x\right\rangle \\
= & \operatorname{Tr}\left[e^{-(\beta \hbar-t) \hat{H} / \hbar} f(x) e^{-\hat{H} t / \hbar}\right] .
\end{aligned}
$$

Using cyclic trace invariance

$$
\begin{aligned}
\langle f[x(t)]\rangle & =\operatorname{Tr}\left[e^{-\beta \hat{H} / \hbar} f(x)\right] \\
& =\langle f(x)\rangle,
\end{aligned}
$$

and the theorem is proved.

Lemma: $\left\langle\bar{f}_{\infty}\right\rangle=\langle f(x)\rangle$.

Proof:

$$
\begin{aligned}
\left\langle\bar{f}_{\infty}\right\rangle & =\left\langle\int_{0}^{1} d u f[x(u)]\right\rangle \\
& =\int_{0}^{1} d u\langle f[x(u)]\rangle .
\end{aligned}
$$

Then from the theorem

$$
\begin{aligned}
\left\langle\bar{f}_{\infty}\right\rangle & =\int_{0}^{1} d u\langle f(x)\rangle \\
& =\langle f(x)\rangle,
\end{aligned}
$$

which proves the lemma.

The equivalence expressed in the lemma is not valid for finite $k_{\max }$, but the result proves point and path estimators provide the same result in the limit that $k_{\max } \rightarrow \infty$.

${ }^{1}$ R. P. Feynman and A. R. Hibbs, Quantum Mechanics and Path Integrals (McGraw-Hill, New York, 1965).

${ }^{2}$ D. M. Ceperley, Rev. Mod. Phys. 67, 279 (1995).

${ }^{3}$ B. J. Berne and D. Thirumalai, Annu. Rev. Phys. Chem. 37, 401 (1986).

${ }^{4}$ D. L. Freeman and J. D. Doll, Adv. Chem. Phys. 70B, 139 (1988).

${ }^{5}$ J. D. Doll, D. L. Freeman, and T. L. Beck, Adv. Chem. Phys. 78, 61 (1990).

${ }^{6}$ N. Makri and W. H. Miller, Chem. Phys. Lett. 151, 1 (1985).

${ }^{7}$ N. Makri and W. H. Miller, J. Chem. Phys. 90, 904 (1989).

${ }^{8}$ R. D. Coalson, D. L. Freeman, and J. D. Doll, J. Chem. Phys. 91, 4242 (1992).

${ }^{9}$ J. Lobaugh and G. A. Voth, J. Chem. Phys. 97, 4205 (1992).

${ }^{10}$ C. Lanczos, Applied Analysis (Dover, New York, 1988), Chap. 4.

${ }^{11}$ J. D. Doll, M. Eleftheriou, S. A. Corcelli, and D. L. Freeman, in Quantum Monte Carlo Methods in Physics and Chemistry, edited by M. P. Nightingale and C. J. Umrigar (Kluwer, Dordrecht, 1999), p. 213.

${ }^{12}$ C. Alexandrou, W. Fleischer, and R. Rosenfelder, Phys. Rev. Lett. 65, 2615 (1990).

${ }^{13}$ J. D. Doll, R. D. Coalson, and D. L. Freeman, Phys. Rev. Lett. 55, 1 (1985).

${ }^{14}$ R. D. Coalson, J. D. Doll, and D. L. Freeman, J. Chem. Phys. 85, 4567 (1986).

${ }^{15}$ M. Abramowitz and I. A. Stegun, Handbook of Mathematical Functions (Dover, New York, 1965), Chap. 23.

${ }^{16}$ D. L. Freeman and J. D. Doll, J. Chem. Phys. 80, 5709 (1984).

${ }^{17}$ M. F. Herman, E. J. Bruskin, and B. J. Berne, J. Chem. Phys. 76, 5150 (1982). 
${ }^{18}$ J. K. Lee, J. A. Barker, and F. F. Abraham, J. Chem. Phys. 58, 3166 (1973).

${ }^{19}$ D. D. Frantz, D. L. Freeman, and J. D. Doll, J. Chem. Phys. 93, 2769 (1990).
${ }^{20}$ D. L. Freeman, R. D. Coalson, and J. D. Doll, J. Stat. Phys. 43, 931 (1986).

${ }^{21}$ C. Chakravarty, M. C. Gordillo, and D. M. Ceperley, J. Chem. Phys. 109, 2123 (1998) 